\title{
Unsymmetrical Diaryl Sulfones and Aryl Vinyl Sulfones through Palladium-Catalyzed Coupling of Aryl and Vinyl Halides or Triflates with Sulfinic Acid Salts
}

\section{Supporting Information}

\author{
Sandro Cacchi, ${ }^{\text {*a }}$ Giancarlo Fabrizi, ${ }^{\text {a }}$ Antonella Goggiamani, ${ }^{\text {a Luca M. Parisi, }{ }^{\text {a }} \text { Roberta Bernini, }}$ \\ ${ }^{a}$ Dipartimento di Studi di Chimica e Tecnologia delle Sostanze Biologicamente Attive \\ Università degli Studi "La Sapienza", P.le A. Moro 5, 00185, Rome, Italy \\ ${ }^{b}$ Dipartimento A.B.A.C., Università degli Studi della Tuscia, Via S. Camillo De Lellis, 01100 Viterbo, Italy
}

\section{Table of contents}

General considerations

Characterization of compounds $\mathbf{3 , 7}$

$2 \mathrm{~S}-4 \mathrm{~S}$

Spectra

$\begin{array}{rr} & 2 S \\ & 2 S-4 S \\ \mathbf{3 g} & \\ \mathbf{3 h} & 5 S-6 S \\ \mathbf{3 i} & 7 \mathrm{~S}-8 \mathrm{~S} \\ \mathbf{3 m} & 9 \mathrm{~S}-10 \mathrm{~S} \\ \mathbf{3 n} & 11 \mathrm{~S}-12 \mathrm{~S} \\ \mathbf{3 o} & 13 \mathrm{~S}-14 \mathrm{~S} \\ \mathbf{3 p} & 15 \mathrm{~S}-16 \mathrm{~S} \\ \mathbf{3 q} & 17 \mathrm{~S}-18 \mathrm{~S} \\ \mathbf{3 r} & 19 \mathrm{~S}-20 \mathrm{~S} \\ \mathbf{3 r} & 21 \mathrm{~S}-22 \mathrm{~S} \\ \mathbf{3 t} & 23 \mathrm{~S}-24 \mathrm{~S} \\ \mathbf{3 u} & 25 \mathrm{~S}-26 \mathrm{~S} \\ \mathbf{3 v} & 27 \mathrm{~S}-28 \mathrm{~S} \\ \mathbf{3 w} & 29 \mathrm{~S}-30 \mathrm{~S} \\ \mathbf{7 b} & 31 \mathrm{~S}-32 \mathrm{~S} \\ \mathbf{7 c} & 33 \mathrm{~S}-34 \mathrm{~S} \\ \mathbf{7 d} & 35 \mathrm{~S}-36 \mathrm{~S} \\ \mathbf{7 e} & 37 \mathrm{~S}-38 \mathrm{~S} \\ \mathbf{7 f} & 39 \mathrm{~S}-40 \mathrm{~S} \\ \mathbf{7 f} & 41 \mathrm{~S}-42 \mathrm{~S}\end{array}$




\section{General considerations}

Melting points were determined with a Büchi apparatus and are uncorrected. Aryl iodides, aryl bromides, bases, salts, solvents, and precatalysts are commercially available and were used as purchased, without further purification. Aryl and vinyl triflates were prepared according to ref.1. Reaction products were purified on axially compressed columns, packed with $\mathrm{SiO}_{2} 25-40 \mu \mathrm{m}$ (Macherey Nagel), connected to a Gilson solvent delivery system and to a Gilson refractive index detector, and eluting with $n$-hexane/ethyl acetate mixtures. Products were crystallized from benzene or dichloromethane/n-hexane mixtures. Unless otherwise stated ${ }^{1} \mathrm{H}$ NMR $(200 \mathrm{MHz})$ and ${ }^{13} \mathrm{C}$ NMR $(50.3 \mathrm{MHz})$ spectra were recorded with a Bruker Avance 200 spectrometer. IR spectra were recorded with a Jasco FT/IR-430 spectrometer. Mass spectra were recorded with VG 70/250S spectrometer with an electron beam of $70 \mathrm{eV}$. Elementary analyses were performed by Carlo Erba 1106 Analyser.

Typical procedure for the preparation of unsymmetrical diaryl sulfones: to a mixture of sodium $p$-toluenesulfinate $(0.075 \mathrm{~g}, 0.420 \mathrm{mmol})$ and $p$-iodoanisole $(0.082 \mathrm{~g}, 0.350 \mathrm{mmol})$ in 2.0 $\mathrm{mL}$ of toluene under argon, $\mathrm{Pd}_{2}(\mathrm{dba})_{3}(0.008 \mathrm{~g}, 0.009 \mathrm{mmol})$, Xantphos (0.010 g, $\left.0.018 \mathrm{mmol}\right)$, $\mathrm{Cs}_{2} \mathrm{CO}_{3}(0.171 \mathrm{~g}, 0.525 \mathrm{mmol})$, and ${ }^{n} \mathrm{Bu}_{4} \mathrm{NCl}(0.117 \mathrm{~g}, 0.420 \mathrm{mmol})$ were added. The mixture was warmed at $80{ }^{\circ} \mathrm{C}$ and stirred for $1 \mathrm{~h}$. After cooling, the reaction mixture was diluted with ethyl acetate, washed with water, dried over $\mathrm{Na}_{2} \mathrm{SO}_{4}$ and concentrated under reduced pressure. The reaction mixture was purified by chromatography (silica gel, $35 \mathrm{~g} ; n$-hexane/ethylacetate $80 / 20 \mathrm{v} / \mathrm{v}$ ) to give $0.0825 \mathrm{~g}$ of $\mathbf{3 a}\left(90 \%\right.$ yield): $\mathrm{mp} 100-1{ }^{\circ} \mathrm{C}$; lit. $\mathrm{mp} 90-91{ }^{\circ} \mathrm{C} ;{ }^{2} \mathrm{IR}(\mathrm{KBr}) 2925,1320,1152 ;{ }^{1} \mathrm{H}$ $\operatorname{NMR}\left(\mathrm{CDCl}_{3}\right) \delta$ 7.57-7.44 (m, 4H), $7.25(\mathrm{~d}, J=8.9 \mathrm{~Hz}, 2 \mathrm{H}), 6.98(\mathrm{~d}, J=8.8 \mathrm{~Hz}, 2 \mathrm{H}) ; 3.86(\mathrm{~s}, 3 \mathrm{H})$; $2.40(\mathrm{~s}, 3 \mathrm{H}) ;{ }^{13} \mathrm{C} \mathrm{NMR}\left(\mathrm{CDCl}_{3}\right) \delta 159.0,138.1,136.4,133.8,129.5,128.0,126.7,114.2,55.4,21.1$. Anal calcd for $\mathrm{C}_{14} \mathrm{H}_{14} \mathrm{O}_{3} \mathrm{~S}$ C, $\mathrm{C}, 64.10 ; \mathrm{H}, 5.38$. Found $\mathrm{C}, 64.01 ; \mathrm{H}, 5.36$.

\section{Characterization of compounds}

3a: mp 100-1 ${ }^{\circ} \mathrm{C}$; Lit. mp $90-91{ }^{\circ} \mathrm{C} ;{ }^{2}$

3b: mp 75-76 ${ }^{\circ} \mathrm{C}$; Lit. mp: $73{ }^{\circ} \mathrm{C} ;{ }^{3}$

3c: mp: $148-9^{\circ} \mathrm{C}$; Lit mp: $158-160^{\circ} \mathrm{C} ;{ }^{4}$

3d: $m p$ 102-3 ${ }^{\circ} \mathrm{C}$; Lit. mp: $115-116{ }^{\circ} \mathrm{C} ;{ }^{5}$

3e: $\mathrm{mp} 119-120^{\circ} \mathrm{C}$; Lit. mp: $113-115^{\circ} \mathrm{C} ;{ }^{6}$

3f: $\mathrm{mp}: 120-1^{\circ} \mathrm{C}$; Lit. mp: $119-122^{\circ} \mathrm{C} ;{ }^{7}$

3g: $\mathrm{mp} \mathrm{85-6}{ }^{\circ} \mathrm{C}$; IR (KBr) 2923, 1323, $1152 \mathrm{~cm}^{-1} ;{ }^{1} \mathrm{H}$ NMR $\left(\mathrm{CDCl}_{3}\right) \delta 8.02-7.87(\mathrm{~m}, 2 \mathrm{H}), 7.80(\mathrm{~d}, J$ $=8.3 \mathrm{~Hz}, 2 \mathrm{H}), 7.28(\mathrm{~d}, J=8.5 \mathrm{~Hz}, 2 \mathrm{H}), 7.20-7.07(\mathrm{~m}, 2 \mathrm{H}), 2.38(\mathrm{~s}, 3 \mathrm{H}) ;{ }^{13} \mathrm{C} \mathrm{NMR}\left(\mathrm{CDCl}_{3}\right) \delta 165.4$ $(\mathrm{d}, J=255.5 \mathrm{~Hz}), 144.5,138.7,130.4(\mathrm{~d}, J=9.5 \mathrm{~Hz}), 130.1,129.6(\mathrm{~d}, J=35.2 \mathrm{~Hz}), 127.7,116.6(\mathrm{~d}$, $J=22.7 \mathrm{~Hz}), 21.6 ;{ }^{19} \mathrm{~F}$ NMR $\{\mathrm{H}\}\left(\mathrm{CDCl}_{3}\right) \delta-109.40 . \mathrm{MS} m / z(\%): 250(60)\left[\mathrm{M}^{+}\right], 139(71), 107$ (84), 91 (72), 65 (100). Anal calcd for $\mathrm{C}_{13} \mathrm{H}_{11} \mathrm{FO}_{2} \mathrm{~S} \mathrm{C}, 62.38 ; \mathrm{H}, 4.43$. Found $\mathrm{C}, 62.30 ; \mathrm{H}, 4.42$.

3h: wax; IR (neat) 2922, 1320, $1154 \mathrm{~cm}^{-1} ;{ }^{1} \mathrm{H}$ NMR $\left(\mathrm{CDCl}_{3}\right) \delta 7.82(\mathrm{~d}, J=8.0 \mathrm{~Hz}, 2 \mathrm{H}), 7.74-7.40$ $(\mathrm{m}, 3 \mathrm{H}), 7.33-7.21(\mathrm{~m}, 3 \mathrm{H}), 2.39(\mathrm{~s}, 3 \mathrm{H}) ;{ }^{13} \mathrm{C} \mathrm{NMR}\left(\mathrm{CDCl}_{3}\right) \delta 162.5(\mathrm{~d}, J=250.5 \mathrm{~Hz}), 144.8$, 138.0, $131.2(\mathrm{~d}, J=7.7 \mathrm{~Hz}), 130.2,127.9,127.7(\mathrm{~d}, J=11.3 \mathrm{~Hz}), 123.4(\mathrm{~d}, J=3.3 \mathrm{~Hz}), 120.5(\mathrm{~d}$, $J=21.3 \mathrm{~Hz}), 114.9(\mathrm{~d}, J=24.3 \mathrm{~Hz}), 21.7 ;{ }^{19} \mathrm{~F} \mathrm{NMR}\{\mathrm{H}\}\left(\mathrm{CDCl}_{3}\right) \delta-109.72 . \mathrm{MS} m / z(\%): 250(54)$ $\left[\mathrm{M}^{+}\right], 139$ (52), 107 (100). Anal calcd for $\mathrm{C}_{13} \mathrm{H}_{11} \mathrm{FO}_{2} \mathrm{~S} \mathrm{C}, 62.38 ; \mathrm{H}, 4.43$. Found $\mathrm{C}, 62.31 ; \mathrm{H}, 4.41$.

3i: $\mathrm{mp} 119-120^{\circ} \mathrm{C}$; Lit. $\mathrm{mp} 115-117^{\circ} \mathrm{C} ;^{5}$

3j: mp $98-9{ }^{\circ} \mathrm{C}$; Lit. mp: $101-2{ }^{\circ} \mathrm{C} ;{ }^{5}$

3k: $\mathrm{mp} 132-3^{\circ} \mathrm{C}$; Lit. $\mathrm{mp} 132-134 ;^{6}$

31: $\mathrm{mp} 87-8{ }^{\circ} \mathrm{C}$; IR $(\mathrm{KBr}) 2983,1725,1324,1155 \mathrm{~cm}^{-1} ;{ }^{1} \mathrm{H}$ NMR $\left(\mathrm{CDCl}_{3}\right) \delta 8.13(\mathrm{~d}, J=8.4 \mathrm{~Hz}$, 2H), $7.97(\mathrm{~d}, J=8.3 \mathrm{~Hz}, 2 \mathrm{H}), 7.82(\mathrm{~d}, J=8.3 \mathrm{~Hz}, 2 \mathrm{H}), 7.30(\mathrm{~d}, J=8.4 \mathrm{~Hz}, 2 \mathrm{H}), 4.37(\mathrm{q}, J=7.1 \mathrm{~Hz}$, 2H), $2.38(\mathrm{~s}, 3 \mathrm{H}), 1.37(\mathrm{t}, J=7.1 \mathrm{~Hz}, 3 \mathrm{H}),{ }^{13} \mathrm{C} \mathrm{NMR}\left(\mathrm{CDCl}_{3}\right) \delta 165.1,145.8,144.8,138.0,134.5$, 130.4, 130.2, 128.0, 127.7, 61.8, 21.7, 14.3. MS m/z (\%): 304 (30) $\left[\mathrm{M}^{+}\right], 139$ (100). Anal calcd for $\mathrm{C}_{16} \mathrm{H}_{16} \mathrm{O}_{4} \mathrm{~S} \mathrm{C}, 63.14 ; \mathrm{H}, 5.30$. Found C, 63.09; H, 5.31. 
3m: $\mathrm{mp} 164-5{ }^{\circ} \mathrm{C}$; IR (KBr) 2926, $1351,1157 \mathrm{~cm}^{-1} ;{ }^{1} \mathrm{H}$ NMR $\left(\mathrm{CDCl}_{3}\right) \delta 8.31(\mathrm{~d}, J=8.9 \mathrm{~Hz}, 2 \mathrm{H})$, $8.10(\mathrm{~d}, J=8.9 \mathrm{~Hz}, 2 \mathrm{H}), 7.84(\mathrm{~d}, J=8.4 \mathrm{~Hz}, 2 \mathrm{H}), 7.34(\mathrm{~d}, J=8.1 \mathrm{~Hz}, 2 \mathrm{H}), 2.41(\mathrm{~s}, 3 \mathrm{H}) ;{ }^{13} \mathrm{C} \mathrm{NMR}$ $\left(\mathrm{CDCl}_{3}\right) \delta 150.3,147.9,145.5,137.1,130.4,128.9,128.2,124.5,21.7$. MS $m / z(\%): 277(34)\left[\mathrm{M}^{+}\right]$, 139 (100). Anal calcd for $\mathrm{C}_{13} \mathrm{H}_{11} \mathrm{NO}_{4} \mathrm{~S}$ C, 56.31; H, 4.00; N, 5.05. Found C, 56.23; H, 3.99; N, 4.98. 3n: $\mathrm{mp} 209-210{ }^{\circ} \mathrm{C}$; IR (KBr) 2957, $1300,1147 \mathrm{~cm}^{-1} ;{ }^{1} \mathrm{H}$ NMR (400 MHz) $\left(\mathrm{CDCl}_{3}\right) \delta 7.80-7.73(\mathrm{~m}$, $4 \mathrm{H}), 7.25(\mathrm{~d}, J=8.0 \mathrm{~Hz}, 2 \mathrm{H}), 6.65(\mathrm{~d}, J=7.1 \mathrm{~Hz}, 2 \mathrm{H}), 3.03(\mathrm{~s}, 6 \mathrm{H}), 2.38(\mathrm{~s}, 3 \mathrm{H}) ;{ }^{13} \mathrm{C} \mathrm{NMR}$ $(100.3 \mathrm{MHz})\left(\mathrm{CDCl}_{3}\right) \delta 153.1,143.1,140.6,129.7,129.4,127.1,127.0,111.1,40.1,21.5 . \mathrm{MS} \mathrm{m} / z$ (\%): $275(91)\left[\mathrm{M}^{+}\right], 136$ (100). Anal calcd for $\mathrm{C}_{15} \mathrm{H}_{17} \mathrm{NO}_{2} \mathrm{~S} \mathrm{C}, 65.43 ; \mathrm{H}, 6.22 ; \mathrm{N}, 5.09$; found $\mathrm{C}$, 65.20; H, 6.24; N, 5.11 .

3o: $\mathrm{mp}: 188-189{ }^{\circ} \mathrm{C}$; IR (KBr) 2923, 1306, $1154 \mathrm{~cm}^{-1} ;{ }^{1} \mathrm{H}$ NMR $\left(\mathrm{CDCl}_{3}\right) \delta 8.02$ (d, $J=8.7 \mathrm{~Hz}, 2$ H), $7.89(\mathrm{~d}, J=6.7 \mathrm{~Hz}, 2 \mathrm{H}), 7.71(\mathrm{~d}, J=8.7 \mathrm{~Hz}, 2 \mathrm{H}), 7.60-7.36(\mathrm{~m}, 5 \mathrm{H}), 7.33(\mathrm{~d}, J=8.0 \mathrm{~Hz}, 2$ $\mathrm{H}), 2.43(\mathrm{~s}, 3 \mathrm{H}) ;{ }^{13} \mathrm{C} \mathrm{NMR}\left(\mathrm{CDCl}_{3}\right) \delta 146.1,145.7,140.4,139.5,137.9,131.6,131.2,131.1,128.8$, 128.2, 127.4, 127.2, 21.5. MS m/z (\%): $308(55)\left[\mathrm{M}^{+}\right], 201(48), 169$ (100), 152 (97). Anal calcd for $\mathrm{C}_{19} \mathrm{H}_{16} \mathrm{O}_{2} \mathrm{~S}, \mathrm{C}, 74.00 ; \mathrm{H}, 5.23$; found $\mathrm{C}, 73.86 ; \mathrm{H}, 5.22$.

3p: mp: $78-79{ }^{\circ} \mathrm{C}$; IR (KBr) 2924, 1317, $1156 \mathrm{~cm}^{-1}$; ${ }^{1} \mathrm{H}$ NMR $\left(\mathrm{CDCl}_{3}\right) \delta 8.61-7.80(\mathrm{~m}, 4 \mathrm{H}), 7.48$ $(\mathrm{d}, J=8.5 \mathrm{~Hz}, 2 \mathrm{H}), 7.27(\mathrm{~d}, J=7.9 \mathrm{~Hz}, 2 \mathrm{H}), 2.37(\mathrm{~s}, 3 \mathrm{H}), 1.28(\mathrm{~s}, 9 \mathrm{H}) ;{ }^{13} \mathrm{C} \mathrm{NMR}\left(\mathrm{CDCl}_{3}\right) \delta$ 156.9, 144.0, 139.1, 139.0, 129.9, 127.7, 127.4, 126.3, 35.2, 31.1, 21.6. MS $m / z(\%): 288(21)\left[\mathrm{M}^{+}\right]$, 273 (100), 139 (81). Anal calcd for $\mathrm{C}_{17} \mathrm{H}_{20} \mathrm{O}_{2} \mathrm{~S}, \mathrm{C}, 70.80 ; \mathrm{H}, 6.99$; found 70.52; H, 6.97.

3q: mp: $139-140{ }^{\circ} \mathrm{C}$; IR ( $\left.\mathrm{KBr}\right) 1315,1153 \mathrm{~cm}^{-1} ;{ }^{1} \mathrm{H}$ NMR $\left(\mathrm{CDCl}_{3}\right) \delta 8.55(\mathrm{~s}, 1 \mathrm{H}), 7.98-7.76(\mathrm{~m}, 6$ $\mathrm{H}), 7.62-7.57(\mathrm{~m}, 2 \mathrm{H}), 7.31(\mathrm{~d}, J=8.8 \mathrm{~Hz}, 2 \mathrm{H}), 2.37(\mathrm{~s}, 3 \mathrm{H}) ;{ }^{13} \mathrm{C} \mathrm{NMR}\left(\mathrm{CDCl}_{3}\right) \delta 144.2,138.9$, $138.8,135.0,132.3,130.0,129.6,129.4,129.1,128.9,128.0,127.8,127.6,122.7,21.6$; MS m/z (\%): $282(86)\left[\mathrm{M}^{+}\right], 139(100), 115$, (89). Anal calcd for $\mathrm{C}_{17} \mathrm{H}_{14} \mathrm{O}_{2} \mathrm{~S}, \mathrm{C}, 72.31 ; \mathrm{H}, 5.00$; found $\mathrm{C}$, $72.53 ; \mathrm{H}, 5.02$.

3r: mp: $136-7{ }^{\circ} \mathrm{C}$; IR (KBr) 2921, 2234, 1593, $1323 \mathrm{~cm}^{-1} ;{ }^{1} \mathrm{H}$ NMR $\left(\mathrm{CDCl}_{3}\right) \delta 8.03(\mathrm{~d}, J=8.6,2$ $\mathrm{H}), 7.84-7.75(\mathrm{~m}, 4 \mathrm{H}), 7.33(\mathrm{~d}, J=8.0 \mathrm{~Hz}, 2 \mathrm{H}), 2.41(\mathrm{~s}, 3 \mathrm{H}) ;{ }^{13} \mathrm{C} \mathrm{NMR}\left(\mathrm{CDCl}_{3}\right) \delta 146.2,145.2$,

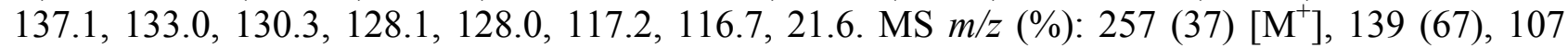
(67), 91 (77), 65 (100). Anal calcd for $\mathrm{C}_{14} \mathrm{H}_{11} \mathrm{NO}_{2} \mathrm{~S}, \mathrm{C}, 65.35 ; \mathrm{H}, 4.31 ; \mathrm{N}, 5.44$; found $\mathrm{C}, 65.68$; $\mathrm{H}$, $4.28 ; \mathrm{N}, 5.34$.

3s: $\mathrm{mp}: 78-79{ }^{\circ} \mathrm{C}$; IR (KBr) 3063, 2236, $1307 \mathrm{~cm}^{-1} ;{ }^{1} \mathrm{H}$ NMR $\left(\mathrm{CDCl}_{3}\right) \delta 8.17(\mathrm{~d}, J=10.1 \mathrm{~Hz}, 2 \mathrm{H})$, 7.86-7.65 (m, $4 \mathrm{H}), 7.35(\mathrm{~d}, J=7.7 \mathrm{~Hz}, 2 \mathrm{H}), 2.43(\mathrm{~s}, 3 \mathrm{H}) ;{ }^{13} \mathrm{C} \mathrm{NMR}\left(\mathrm{CDCl}_{3}\right) \delta 145.3,143.9$,

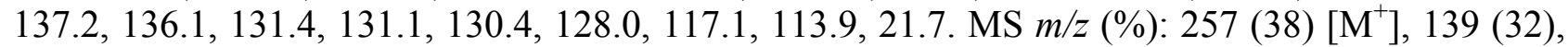
107 (100), 91 (96), 65 (99). Anal calcd for $\mathrm{C}_{14} \mathrm{H}_{11} \mathrm{NO}_{2} \mathrm{~S}, \mathrm{C}, 65.35 ; \mathrm{H}, 4.31 ; \mathrm{N}, 5.44$; found $\mathrm{C}, 65.22$; H, 4.29; N, 5.41 .

3t: $\mathrm{mp}: 131-132{ }^{\circ} \mathrm{C}$; IR (KBr) 1351, $1327,1130 \mathrm{~cm}^{-1} ;{ }^{1} \mathrm{H}$ NMR $\left(\mathrm{CDCl}_{3}\right) \delta 8.75-8.73(\mathrm{~m}, 1 \mathrm{H}), 8.42-$ $8.37(\mathrm{~m}, 1 \mathrm{H}), 8.30-8.21(\mathrm{~m}, 1 \mathrm{H}), 7.86(\mathrm{~d}, J=8.3 \mathrm{~Hz}, 2 \mathrm{H}), 7.72(\mathrm{t}, J=8.1 \mathrm{~Hz}, 1 \mathrm{H}), 7.34(\mathrm{~d}, J=$ 8.3, $2 \mathrm{H}), 2.42(\mathrm{~s}, 3 \mathrm{H}) ;{ }^{13} \mathrm{C} \mathrm{NMR}\left(\mathrm{CDCl}_{3}\right) \delta 145.4,144.5,137.2,133.0,130.7,130.4,128.1,128.0$, 127.5, 122.9, 21.7. MS m/z (\%): 277 (31) $\left[\mathrm{M}^{+}\right], 139$ (28), 107 (91), 91 (100), 65 (87). Anal calcd for $\mathrm{C}_{13} \mathrm{H}_{11} \mathrm{NO}_{4} \mathrm{~S}, \mathrm{C}, 56.31 ; \mathrm{H}, 4.00 ; \mathrm{N}, 5.05$; found $\mathrm{C}, 56.14 ; \mathrm{H}, 4.03 ; \mathrm{N}, 5.07$.

3u: mp: $147-148{ }^{\circ} \mathrm{C}$; IR (KBr) 1706, $1321,1155 \mathrm{~cm}^{-1} ;{ }^{1} \mathrm{H}$ NMR $\left(\mathrm{CDCl}_{3}\right) \delta 10.09(\mathrm{~s}, 1 \mathrm{H}), 8.14-7.99$ $(\mathrm{m}, 4 \mathrm{H}), 7.87(\mathrm{~d}, J=8.3 \mathrm{~Hz}, 2 \mathrm{H}), 7.37(\mathrm{~d}, J=8.3 \mathrm{~Hz}, 2 \mathrm{H}), 2.43(\mathrm{~s}, 3 \mathrm{H}) ;{ }^{13} \mathrm{C} \mathrm{NMR}\left(\mathrm{CDCl}_{3}\right) \delta$ $190.8,147.1,145.0,139.0,137.6,130.3,130.2,128.2,128.0,21.6 . \mathrm{MS} m / z(\%): 260(36)\left[\mathrm{M}^{+}\right], 139$ (80), 107 (81), 91 (94), 65 (100). Anal calcd for $\mathrm{C}_{14} \mathrm{H}_{12} \mathrm{O}_{3} \mathrm{~S}, \mathrm{C}, 64.60 ; \mathrm{H}, 4.65$; found $\mathrm{C}, 64.34$; $\mathrm{H}$, 4.67 .

3v: $\mathrm{mp}: 102-103{ }^{\circ} \mathrm{C}$; IR (KBr) 1317, $1157 \mathrm{~cm}^{-1} ;{ }^{1} \mathrm{H}$ NMR $\left(\mathrm{CDCl}_{3}\right) \delta 8.67-8.64(\mathrm{~m}, 1 \mathrm{H}), 8.18(\mathrm{~d}, J$ $=7.8 \mathrm{~Hz}, 1 \mathrm{H}), 7.97-7.90(\mathrm{~m}, 3 \mathrm{H}), 7.48-7.43(\mathrm{~m}, 1 \mathrm{H}), 7.33(\mathrm{~d}, J=8.2 \mathrm{~Hz}, 2 \mathrm{H}), 2.41(\mathrm{~s}, 3 \mathrm{H}) ;{ }^{13} \mathrm{C}$ NMR $\left(\mathrm{CDCl}_{3}\right) \delta 159.3,150.4,144.8,138.0,135.9,129.7,129.0,126.7,122.0,21.7 . \mathrm{MS} \mathrm{m} / z(\%)$ : 169 (100) [M-64]. Anal calcd for $\mathrm{C}_{12} \mathrm{H}_{11} \mathrm{NO}_{2} \mathrm{~S}, \mathrm{C}, 61.78 ; \mathrm{H}, 4.75 ; \mathrm{N}, 6.00$; found $\mathrm{C}, 62.09 ; \mathrm{H}, 4.73$; N, 5.98.

3w: $140-1{ }^{\circ} \mathrm{C}$; IR (KBr) 3048, 2933, 1309, $1159 \mathrm{~cm}^{-1} ;{ }^{1} \mathrm{H}$ NMR $(400 \mathrm{MHz})\left(\mathrm{CDCl}_{3}\right) \delta 8.37(\mathrm{~d}, J=$ $8.5 \mathrm{~Hz}, 1 \mathrm{H}), 8.23-8.16(\mathrm{~m}, 3 \mathrm{H}), 8.03(\mathrm{~d}, J=8.3 \mathrm{~Hz}, 2 \mathrm{H}), 7.88(\mathrm{~d}, J=8.3 \mathrm{~Hz}, 1 \mathrm{H}), 7.79\left(\mathrm{dt}, J_{1}=\right.$ 
$\left.6.9 \mathrm{~Hz}, J_{2}=1.2 \mathrm{~Hz}, 1 \mathrm{H}\right), 7.66\left(\mathrm{dt}, J_{1}=6.9 \mathrm{~Hz}, J_{2}=1.2 \mathrm{~Hz}, 1 \mathrm{H}\right), 7.34(\mathrm{~d}, J=8.1 \mathrm{~Hz}, 2 \mathrm{H}), 2.41(\mathrm{~s}$, $3 \mathrm{H}) ;{ }^{13} \mathrm{C}$ NMR $(100.3 \mathrm{MHz})\left(\mathrm{CDCl}_{3}\right) \delta 158.5,147.5,144.9,138.7,136.3,131.0,130.5,129.8$, 129.2, 128.9, 127.8, 117.8, 21.7. MS m/z (\%): 219 (100), 204 (52), 128 (48). Anal calcd for $\mathrm{C}_{16} \mathrm{H}_{13} \mathrm{NO}_{2} \mathrm{~S} \mathrm{C}, 67.82 ; \mathrm{H}, 4.62 ; \mathrm{N}, 4.94$; found $\mathrm{C}, 67.62 ; \mathrm{H}, 4.60 ; \mathrm{N}, 4.92$.

7a: mp: $123-124{ }^{\circ} \mathrm{C}$; Lit. mp: $126-127{ }^{\circ} \mathrm{C} .{ }^{8}$

7b: $\mathrm{mp}$ 61-63 ${ }^{\circ} \mathrm{C}$; IR (KBr) 2927, 2850, 1287, $1147 \mathrm{~cm}^{-1} ;{ }^{1} \mathrm{H}$ NMR (400 MHz) $\left(\mathrm{CDCl}_{3}\right) \delta 7.76(\mathrm{~d}, J$ $=8.3 \mathrm{~Hz}, 2 \mathrm{H}), 7.32(\mathrm{~d}, J=8.3 \mathrm{~Hz}, 2 \mathrm{H}), 7.09(\mathrm{t}, J=8.4 \mathrm{~Hz}, 1 \mathrm{H}), 2.44(\mathrm{~s}, 3 \mathrm{H}), 2.43-2.37(\mathrm{~m}, 2 \mathrm{H})$, 2.35-2.28 (m, $2 \mathrm{H}), 1.68-1.62(\mathrm{~m}, 2 \mathrm{H}), 1.42-1.39(\mathrm{~m}, 6 \mathrm{H}) ;{ }^{13} \mathrm{C}$ NMR $(100.3 \mathrm{MHz})\left(\mathrm{CDCl}_{3}\right) \delta$ 144.0, 142.1, 140.8, 137.0, 129.8, 128.3, 29.2, 28.3, 26.6, 25.9, 25.7, 25.2, 21.7. MS m/z (\%): 264 (5) $\left[\mathrm{M}^{+}\right], 91$ (27), 67 (100). Anal calcd for $\mathrm{C}_{15} \mathrm{H}_{20} \mathrm{O}_{2} \mathrm{~S} \mathrm{C}, 68.14 ; \mathrm{H}, 7.62$; found $\mathrm{C}, 68.01 ; \mathrm{H}, 7.64$.

7c: $\mathrm{mp}: 121-122{ }^{\circ} \mathrm{C}$; IR (KBr) 2957, 1311, 1288, $1149 \mathrm{~cm}^{-1} ;{ }^{1} \mathrm{H}$ NMR $(400 \mathrm{MHz})\left(\mathrm{CDCl}_{3}\right) \delta 7.75$ $(\mathrm{d}, J=8.2 \mathrm{~Hz}, 2 \mathrm{H}), 7.33(\mathrm{~d}, J=8.2 \mathrm{~Hz}, 2 \mathrm{H}), 7.06-7.02(\mathrm{~m}, 1 \mathrm{H}), 2.44(\mathrm{~s}, 3 \mathrm{H}), 2.43-2.32(\mathrm{~m}, 2 \mathrm{H})$, 2.08-1.86 (m, $3 \mathrm{H}), 1.26-1.10(\mathrm{~m}, 2 \mathrm{H}), 0.87(\mathrm{~s}, 9 \mathrm{H}) ;{ }^{13} \mathrm{C} \mathrm{NMR}(100.3 \mathrm{MHz})\left(\mathrm{CDCl}_{3}\right) \delta 144.0$, 140.0, 138.5, 136.7, 129.8, 128.1, 42.9, 32.2, 27.3, 27.1, 24.4, 23.5, 21.7. MS m/z (\%): 292 (12) $\left[\mathrm{M}^{+}\right.$], 236 (58), 139 (32), 91 (40), 57 (100). Anal calcd for $\mathrm{C}_{17} \mathrm{H}_{24} \mathrm{O}_{2} \mathrm{~S} \mathrm{C}, 69.82 ; \mathrm{H}, 8.27$; found $\mathrm{C}$, $69.54 ; \mathrm{H}, 8.25$.

7d: $\mathrm{mp}$ 122-123 ${ }^{\circ} \mathrm{C}$; IR (KBr) 2954, 2918, 2866, 1299, $1143 \mathrm{~cm}^{-1} ;{ }^{1} \mathrm{H}$ NMR $(400 \mathrm{MHz})\left(\mathrm{CDCl}_{3}\right) \delta$ $7.43(\mathrm{~d}, J=8.3 \mathrm{~Hz}, 2 \mathrm{H}), 7.32(\mathrm{~d}, J=8.2 \mathrm{~Hz}, 2 \mathrm{H}), 6.82(\mathrm{~s}, 1 \mathrm{H}), 2.44(\mathrm{~s}, 3 \mathrm{H}), 1.88(\mathrm{~s}, 2 \mathrm{H}), 1.32$ $(\mathrm{s}, 2 \mathrm{H}), 1.11(\mathrm{~s}, 6 \mathrm{H}), 0.85(\mathrm{~s}, 6 \mathrm{H}) ;{ }^{13} \mathrm{C} \mathrm{NMR}(100.3 \mathrm{MHz})\left(\mathrm{CDCl}_{3}\right) \delta 145.4,144.0,136.5,136.4$,

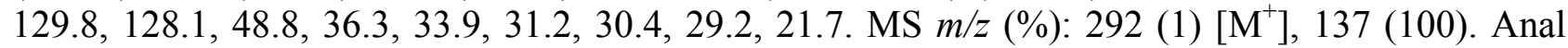
calcd for $\mathrm{C}_{17} \mathrm{H}_{24} \mathrm{O}_{2} \mathrm{~S} \mathrm{C}, 69.82 ; \mathrm{H}, 8.27$; found $\mathrm{C}, 70.31 ; \mathrm{H}, 8.24$.

7e: $\mathrm{mp}$ 200-201 ${ }^{\circ} \mathrm{C}$; IR (KBr): 2944, 1738, 1313, $1143 \mathrm{~cm}^{-1} ;{ }^{1} \mathrm{H}$ NMR $(400 \mathrm{MHz})\left(\mathrm{CDCl}_{3}\right) \delta 7.76$ $(\mathrm{d}, J=7.8 \mathrm{~Hz}, 2 \mathrm{H}), 7.32(\mathrm{~d}, J=7.8 \mathrm{~Hz}, 2 \mathrm{H}), 5.95-5.92(\mathrm{~m}, 1 \mathrm{H}), 4.62(\mathrm{t}, J=8.5 \mathrm{~Hz}, 1 \mathrm{H}), 2.77(\mathrm{~s}$, $3 \mathrm{H}), 2.39-2.04(\mathrm{~m}, 4 \mathrm{H}), 2.06(\mathrm{~m}, 3 \mathrm{H}), 1.92-0.95(\mathrm{~m}, 14 \mathrm{H}), 0.86(\mathrm{~s}, 3 \mathrm{H}), 0.83(\mathrm{~s}, 3 \mathrm{H}) ;{ }^{13} \mathrm{C} \mathrm{NMR}$ $(100.3 \mathrm{MHz})\left(\mathrm{CDCl}_{3}\right) \delta 171.3,144.0,139.7,137.0,136.6,134.8,133.9,129.8,127.9,82.6,51.2$, 47.8, 42.6, 36.7, 34.9, 33.4, 32.0, 31.5, 27.6, 23.5, 21.7, 21.2, 20.9, 20.7, 19.0, 12.1. Anal calcd for $\mathrm{C}_{28} \mathrm{H}_{36} \mathrm{O}_{4} \mathrm{~S}$ C, 71.76; H, 7.74; found C, 71.62; H, 7.71.

7f: mp: $178-179{ }^{\circ} \mathrm{C}$; IR (KBr): 2929, 1701, 1313, $1151 \mathrm{~cm}^{-1} ;{ }^{1} \mathrm{H}$ NMR $(400 \mathrm{MHz})\left(\mathrm{CDCl}_{3}\right) \delta 7.76$ $(\mathrm{d}, J=8.2 \mathrm{~Hz}, 2 \mathrm{H}), 7.32(\mathrm{~d}, J=8.2 \mathrm{~Hz}, 2 \mathrm{H}), 7.10-7.08(\mathrm{~m}, 1 \mathrm{H}), 5.96-5.92(\mathrm{~m}, 1 \mathrm{H}), 2.58-0.99$ (m, $18 \mathrm{H}), 2.44(\mathrm{~s}, 3 \mathrm{H}), 2.13(\mathrm{~s}, 3 \mathrm{H}), 0.83(\mathrm{~s}, 3 \mathrm{H}), 0.66(\mathrm{~s}, 3 \mathrm{H}) \cdot{ }^{13} \mathrm{C} \mathrm{NMR}(100.3 \mathrm{MHz})\left(\mathrm{CDCl}_{3}\right) \delta$ 209.3, 144.0, 139.6, 137.0, 136.6, 134.8, 134.0, 130.0, 127.9, 63.6, 56.9, 47.7, 44.0, 38.7, 34.8, $33.4,32.2,31.6,31.5,24.4,22.9,21.7,21.1,20.9,19.0,13.4$. Anal calcd for $\mathrm{C}_{28} \mathrm{H}_{36} \mathrm{O}_{3} \mathrm{~S}$ C, 74.30 ; $\mathrm{H}, 8.02$; found $\mathrm{C}, 74.08 ; \mathrm{H}, 8.00$.

\footnotetext{
${ }^{1}$ (a) Stang, P. G.; Treptow, W. Synthesis 1980, 283. (b) Stang, P. G.; Hanack, M.; Subramanian, L. R. Synthesis 1982, 85.

${ }^{2}$ El-Khawaga, A. M.; Roberts, R. M. J. Org. Chem. 1985, 50, 3334.

${ }^{3}$ Neumann, W. P.; Wicenec, C. Chem. Ber. 1993, 126), 763.

${ }^{4}$ Ueda, M.; Uchiyama, K.; Kano, T. Synthesis 1984, 4, 323.

${ }^{5}$ Campbell, J. R.; Hatton, R. E. J. Org. Chem. 1961, 26, 2480.

${ }^{6}$ Singh, R. P.; Kamble, R. M.; Chandra, K. L.; Saravanan P.; Singh, V. K. Tetrahedron, 2001, 57, 241 and references therein.

${ }^{7}$ Baarschers, W. H. Can. J. Chem. 1976, 54, 3056.

${ }^{8}$ Inomata, K.; Kinoshita, H.; Takemoto, H.; Murata, Y; Kotake, H. Bull. Chem. Soc. Japan, 1978, $51,3341$.
} 
3g
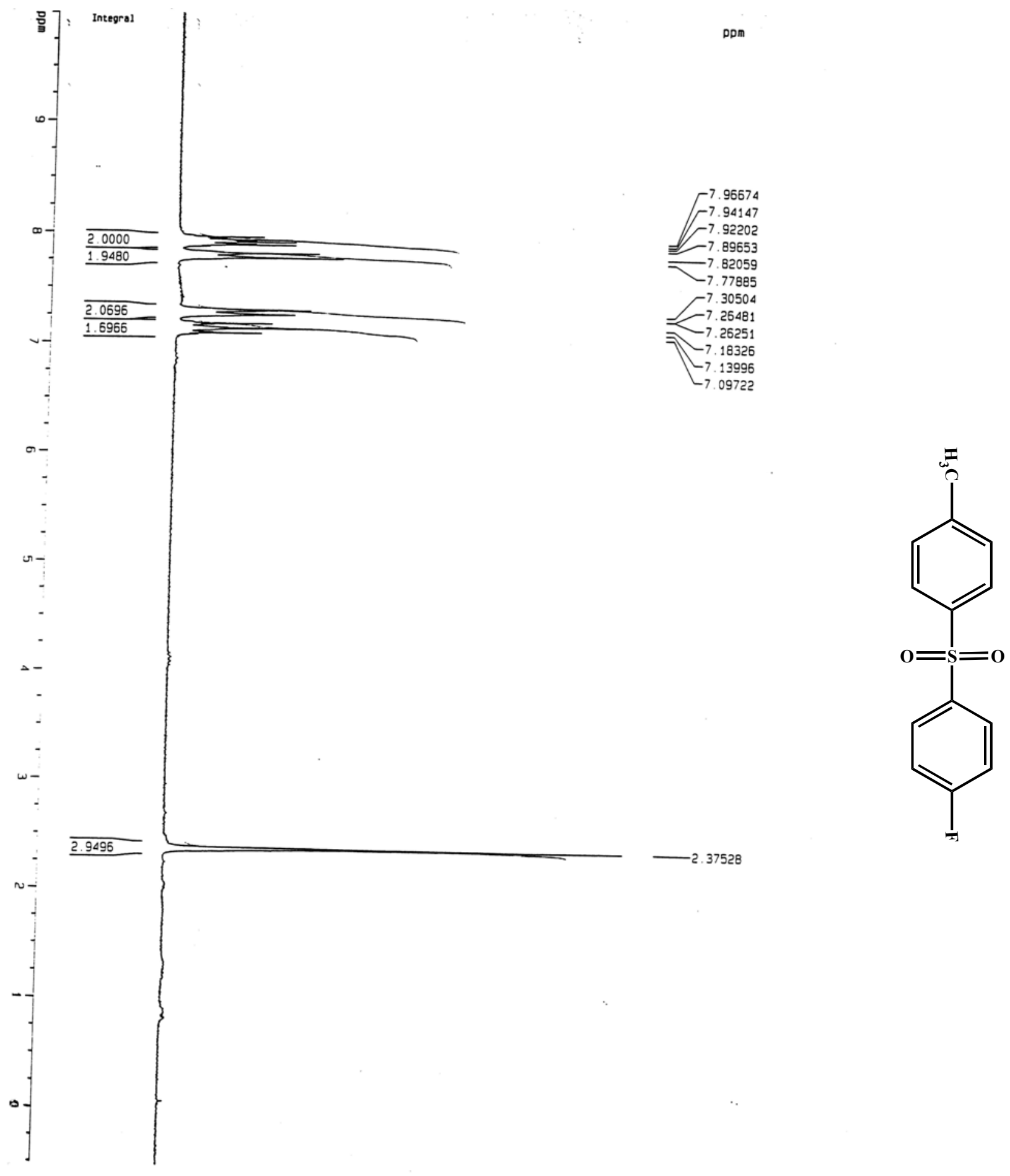


$$
1
$$


3h

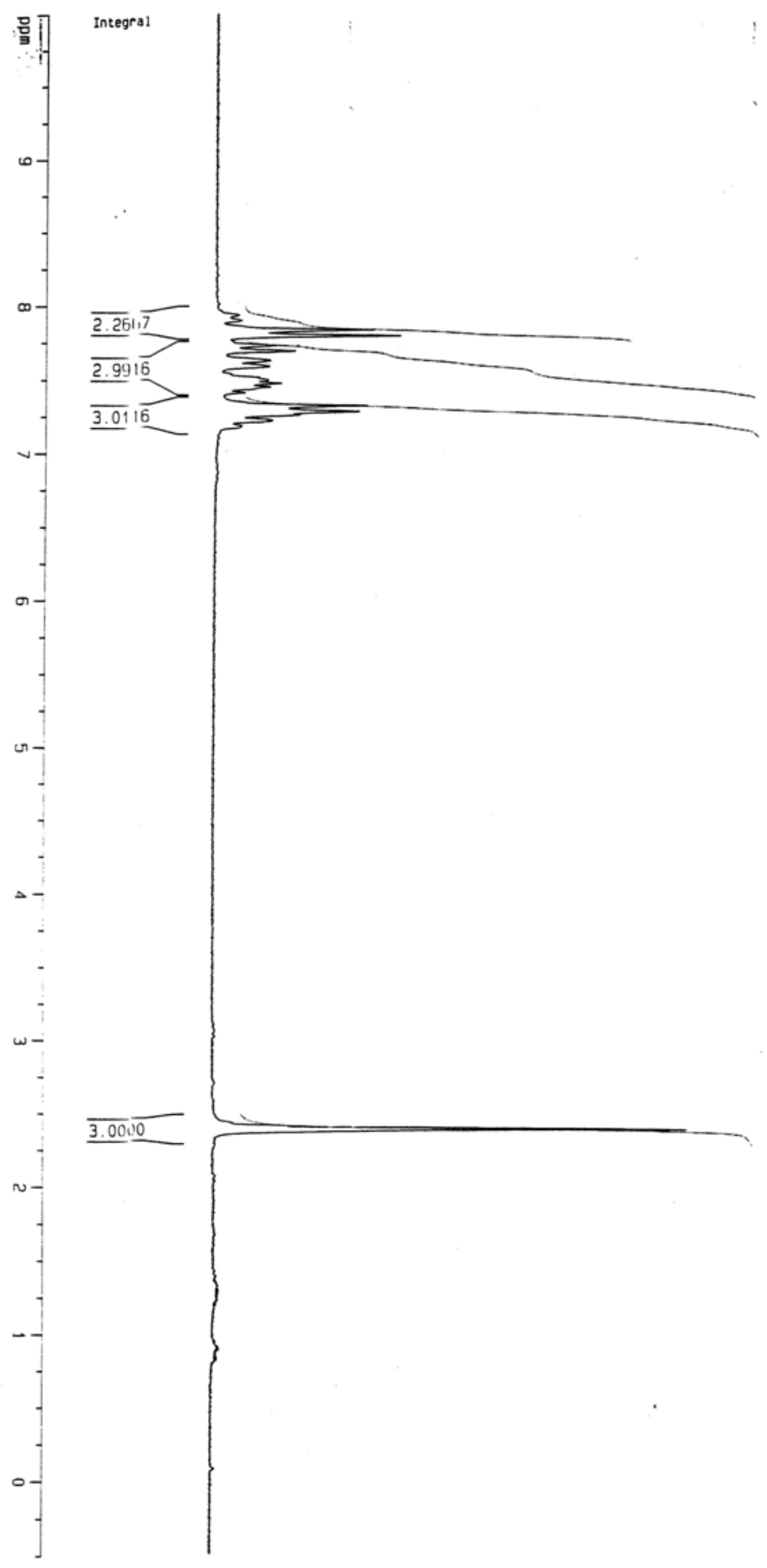

ppm

-7.83870
-7.79849

-7.32572
-7.28612

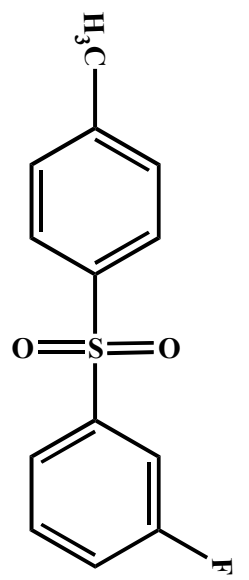




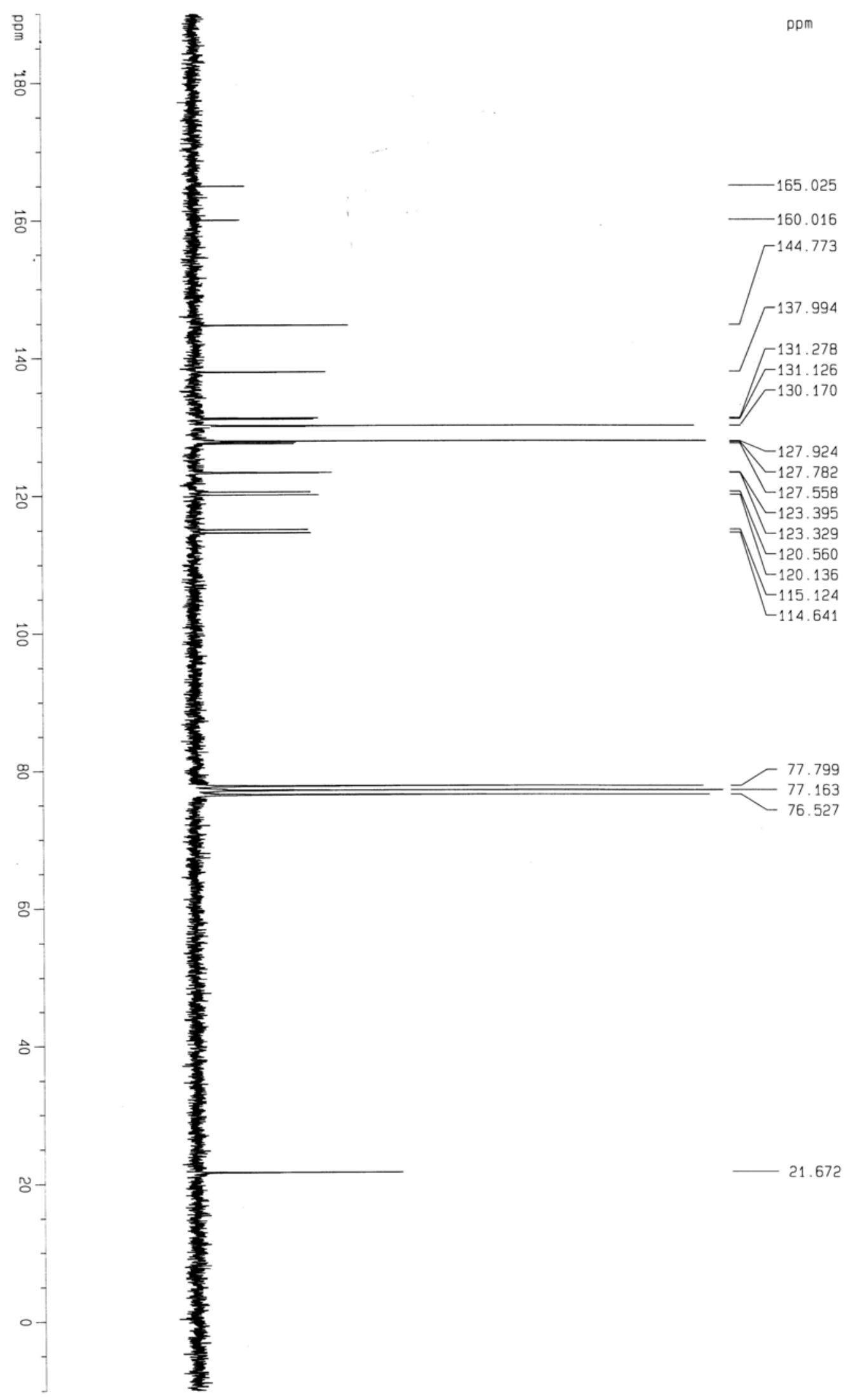


31
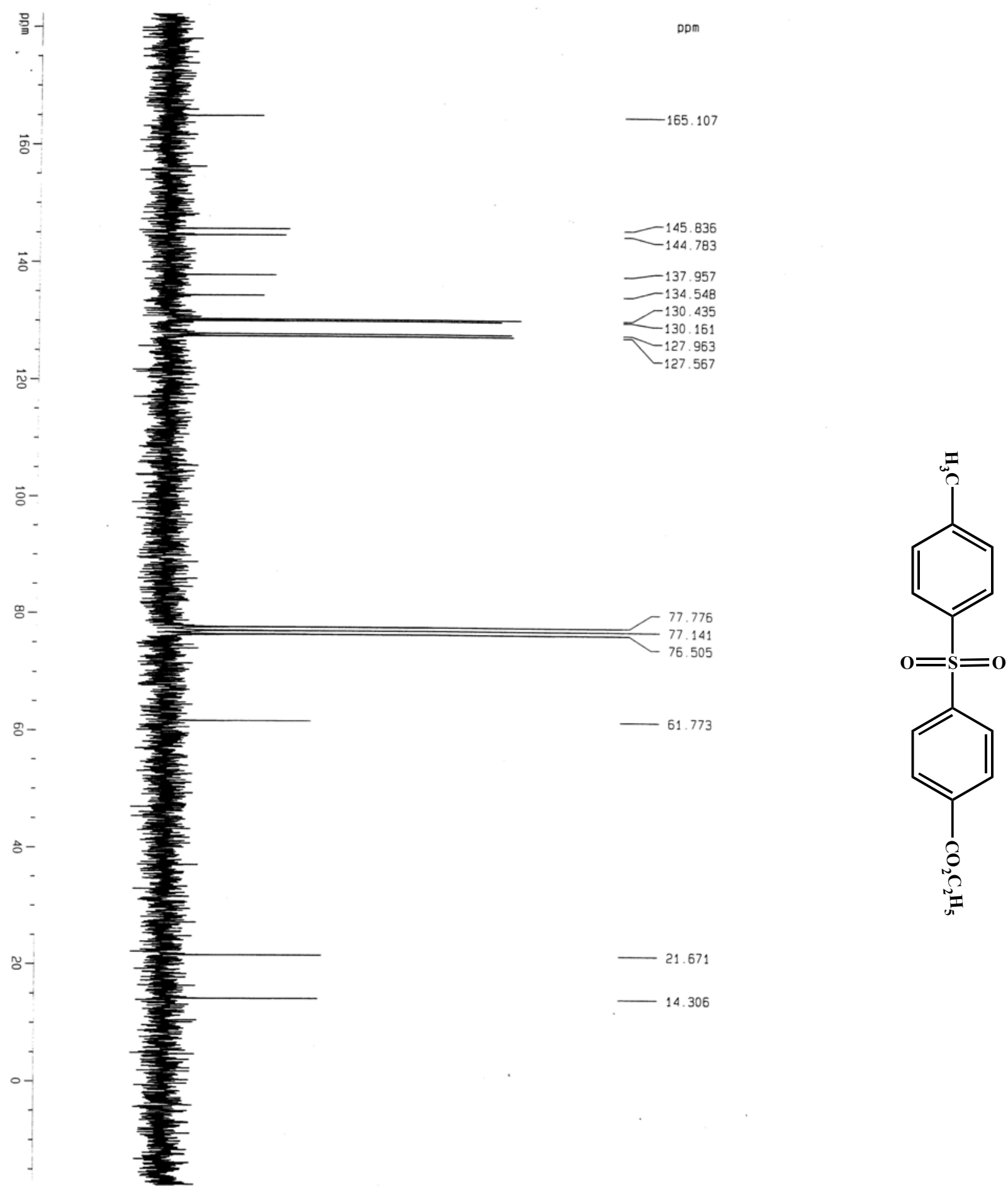


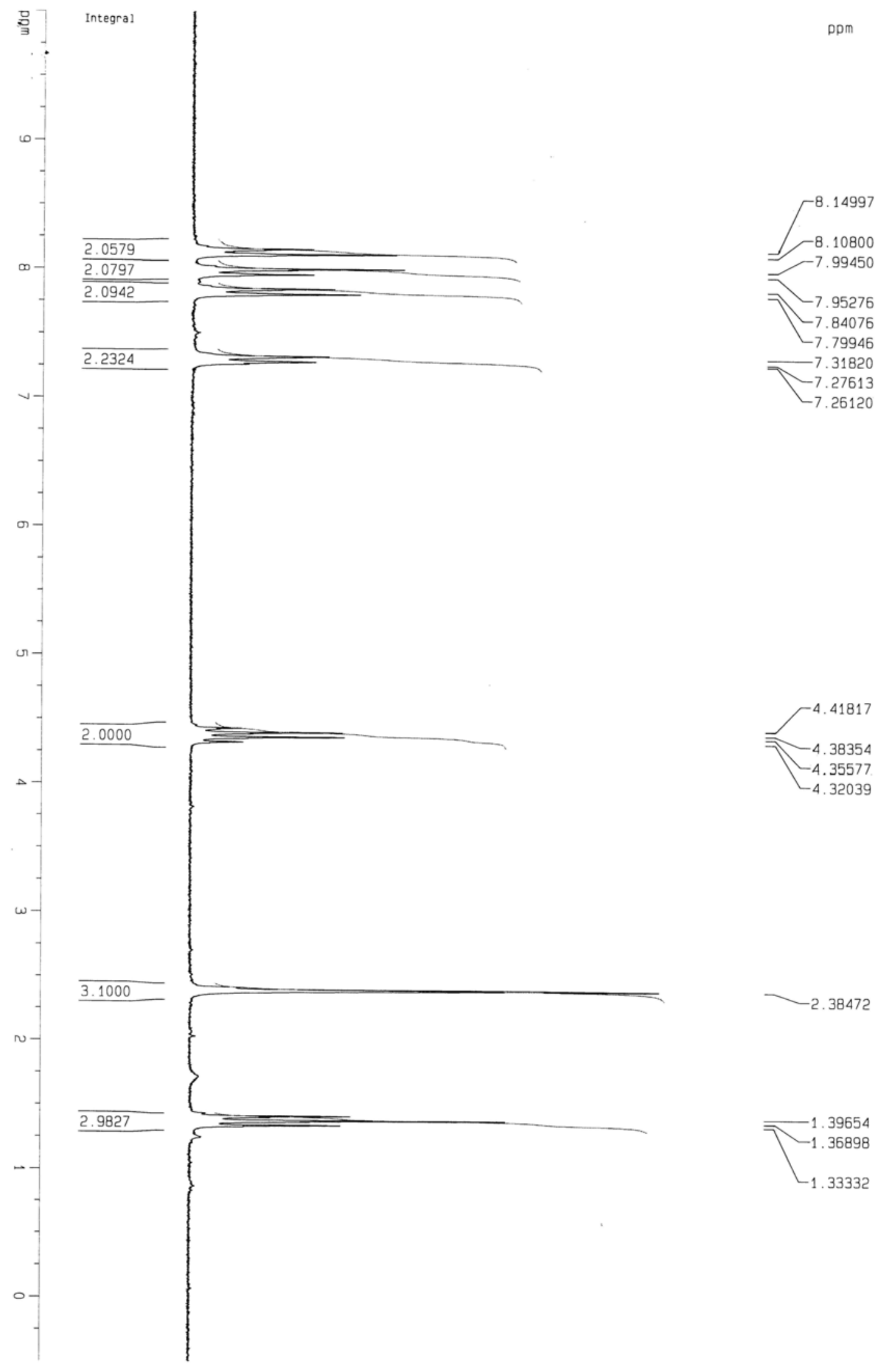


- 11S -

$3 m$
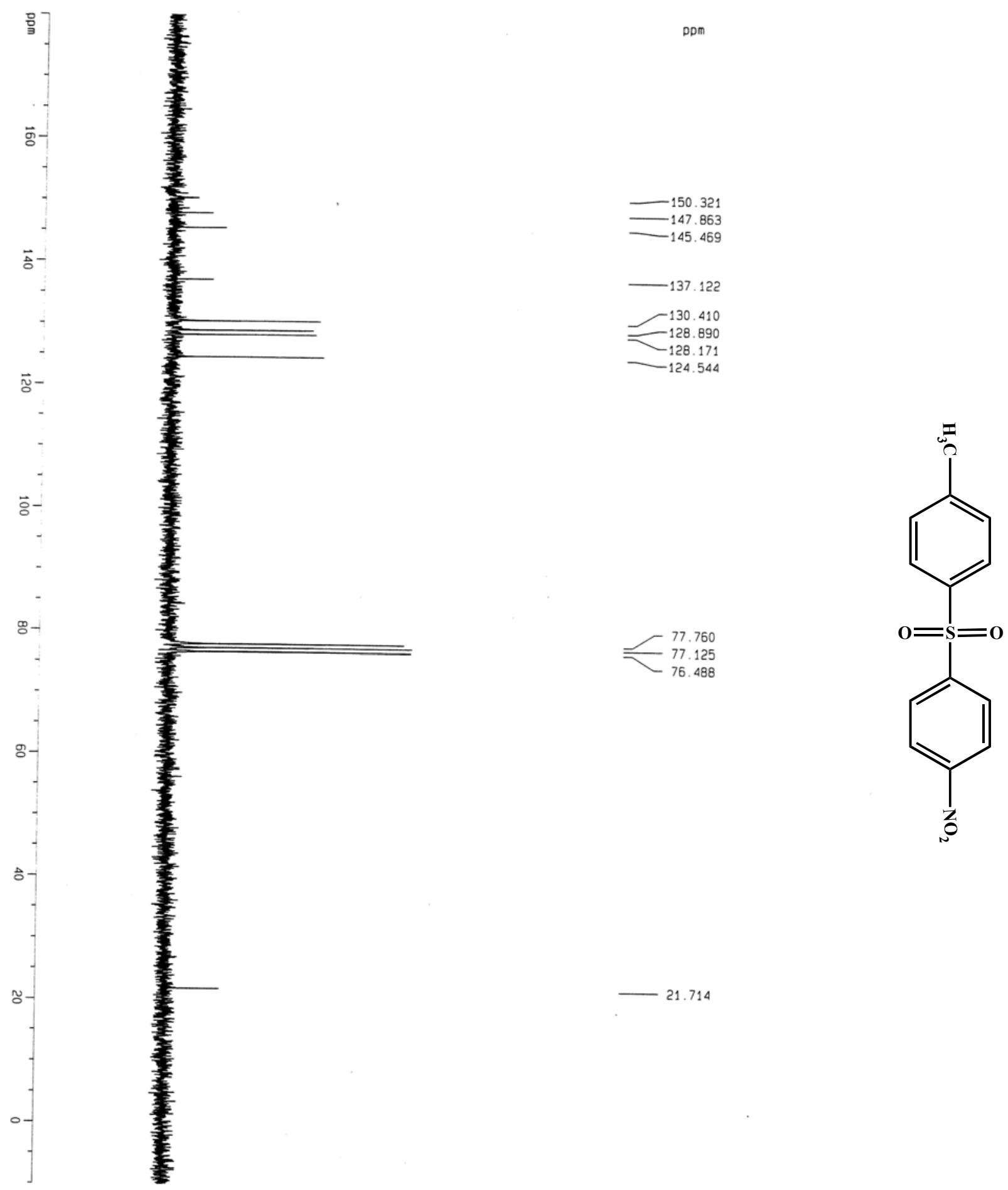


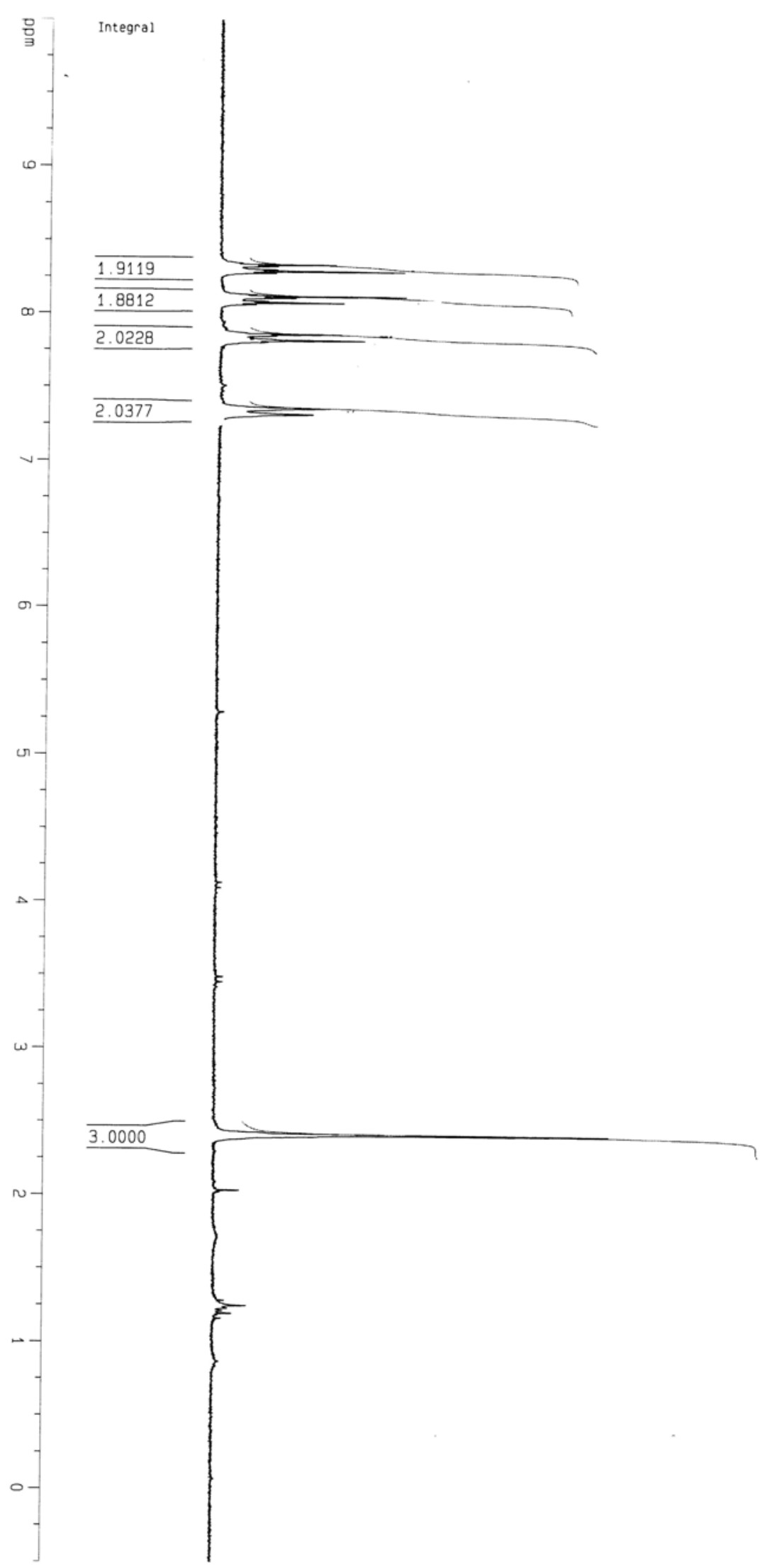


3n

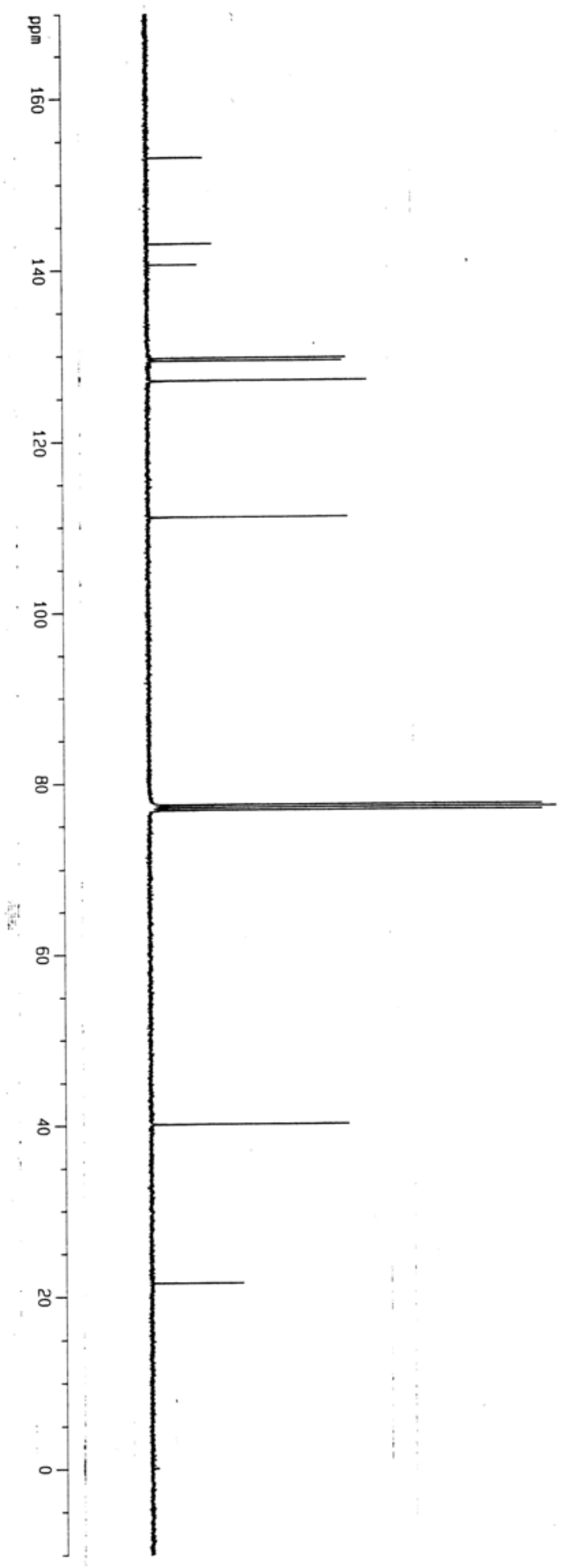

ppm

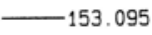

$-143.052$

40.617

$-129.702$

$\checkmark 127.069$

$-111.145$
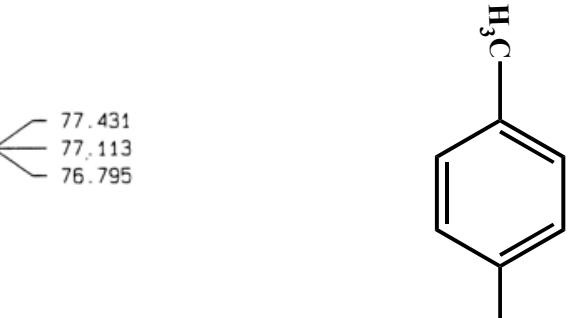

$-21.526$

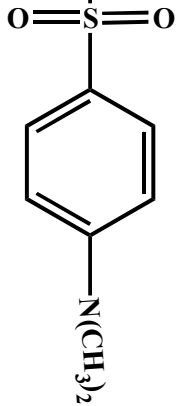


- 14S -

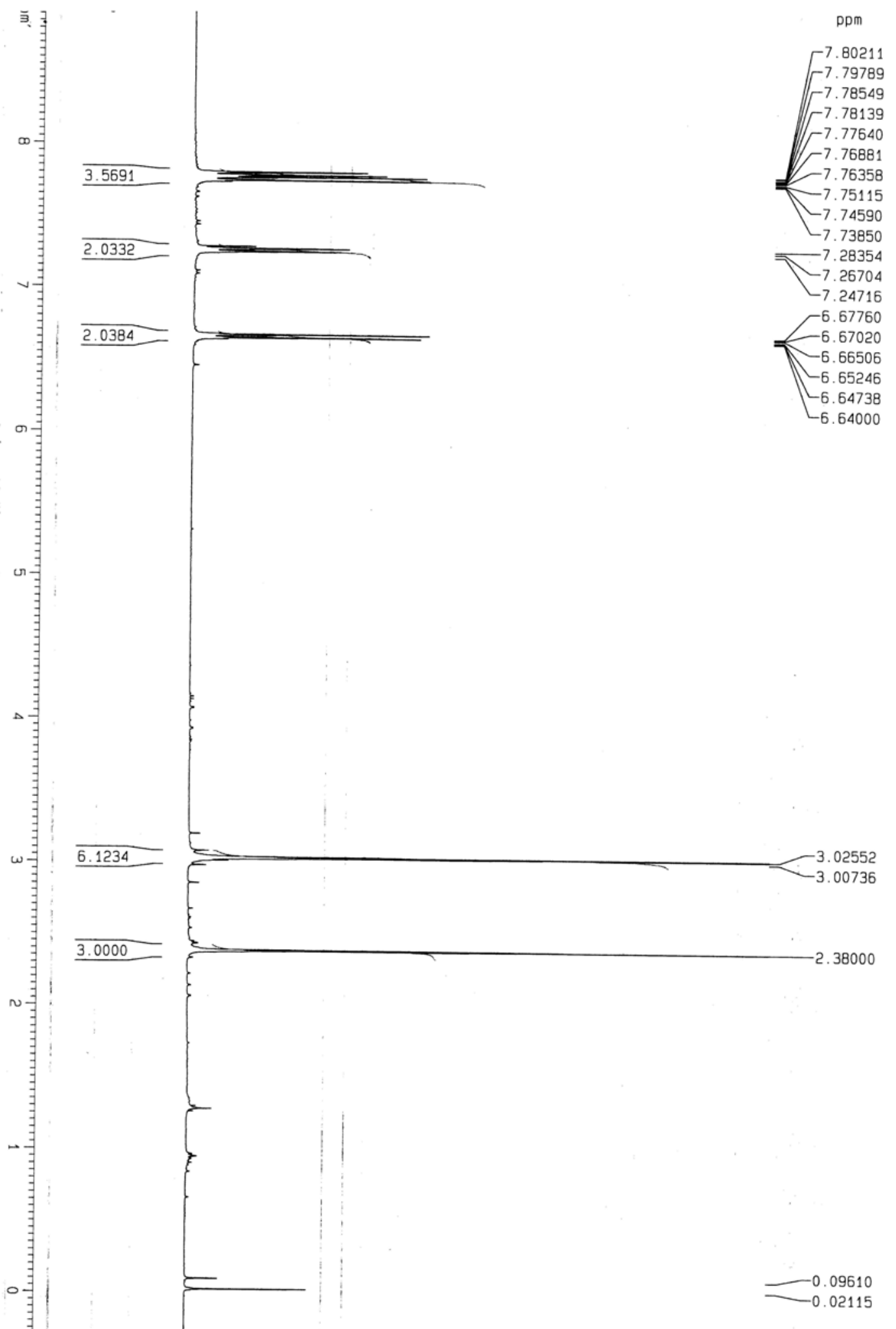


30

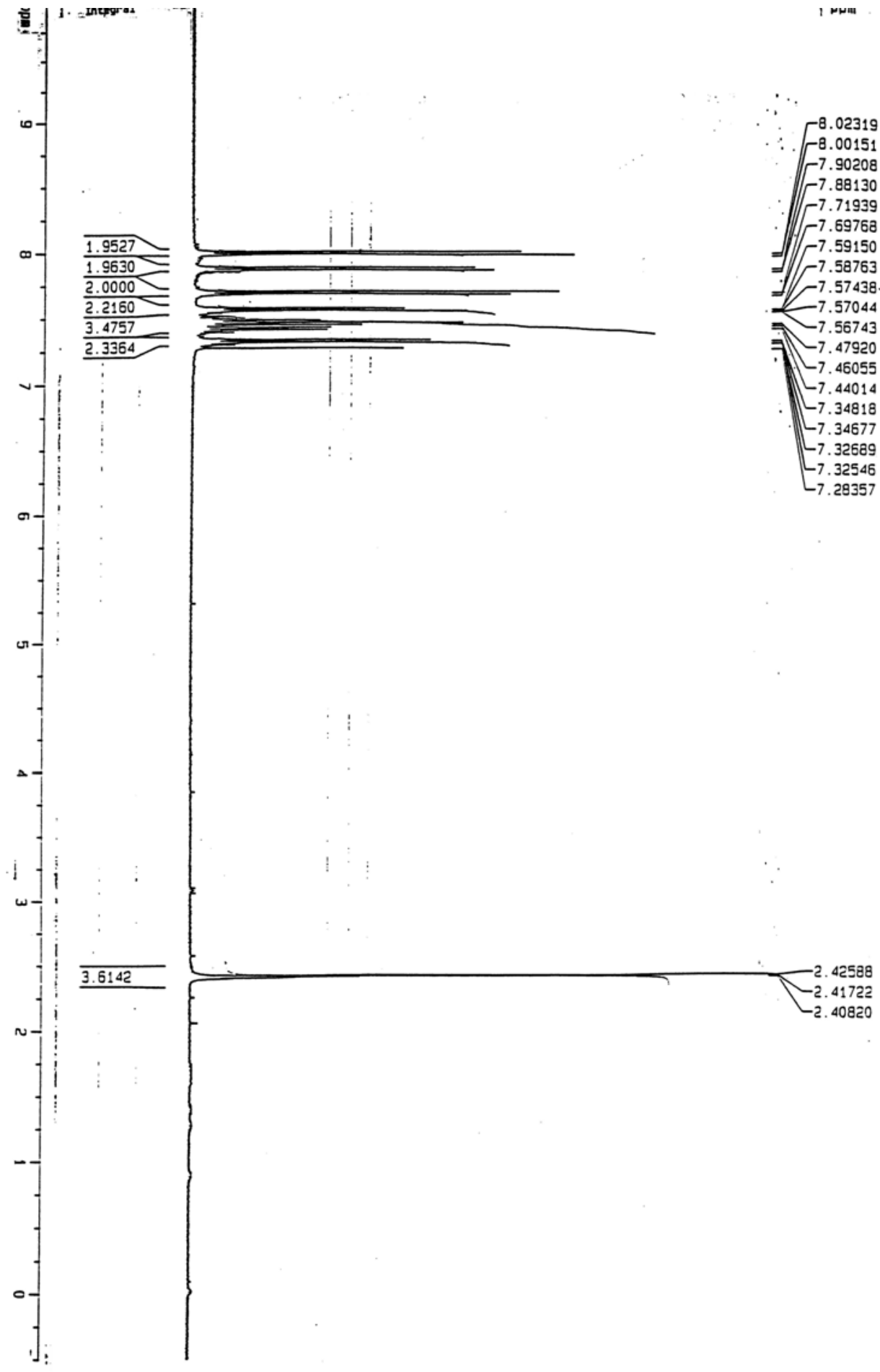


$-16 \mathrm{~S}-$

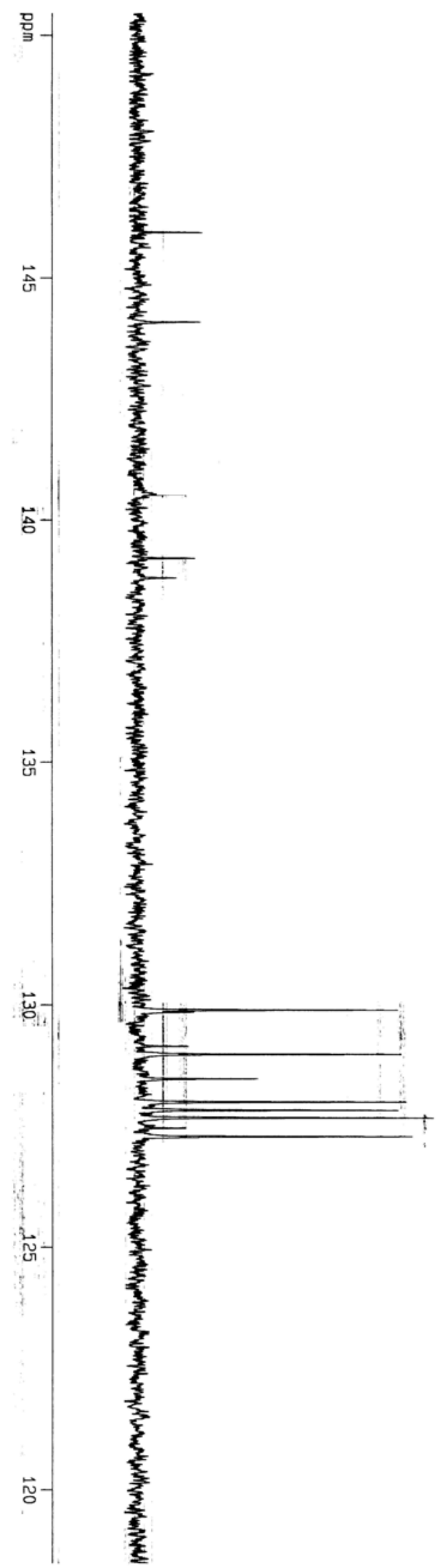

ppm

$-145.948$

$-144.092$

$-140.538$

$-139.210$

138.81

$-129.891$

$-129.848$

$-129.146$

128.978

$-127.988$

$-127.822$

$-127.666$

$-127.457$

$-127.281$ 
$3 p$
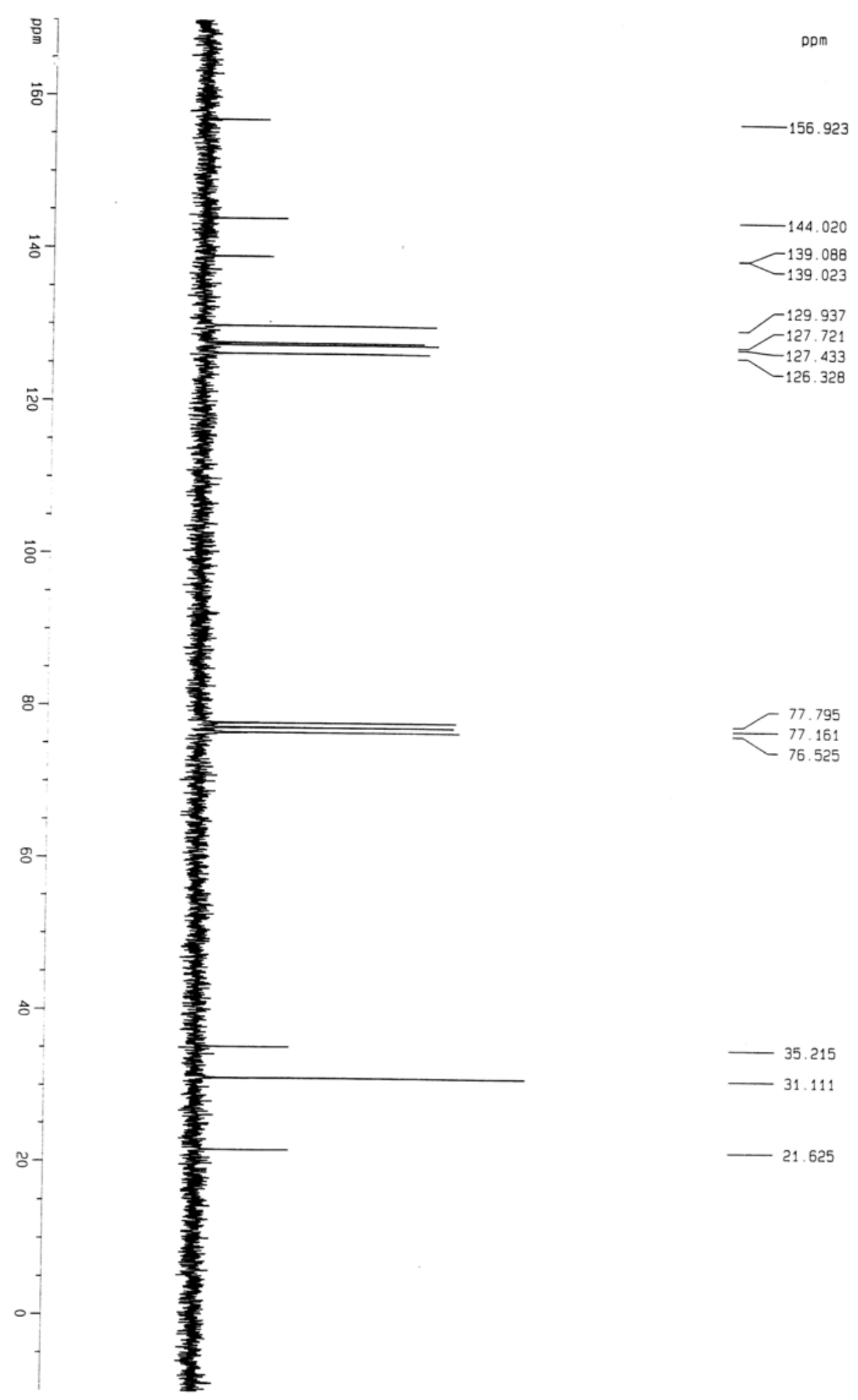<smiles>COc1ccc(S(=O)(=O)c2ccc([O-])cc2)cc1</smiles>

- 35.215 31.111 
- 18S -

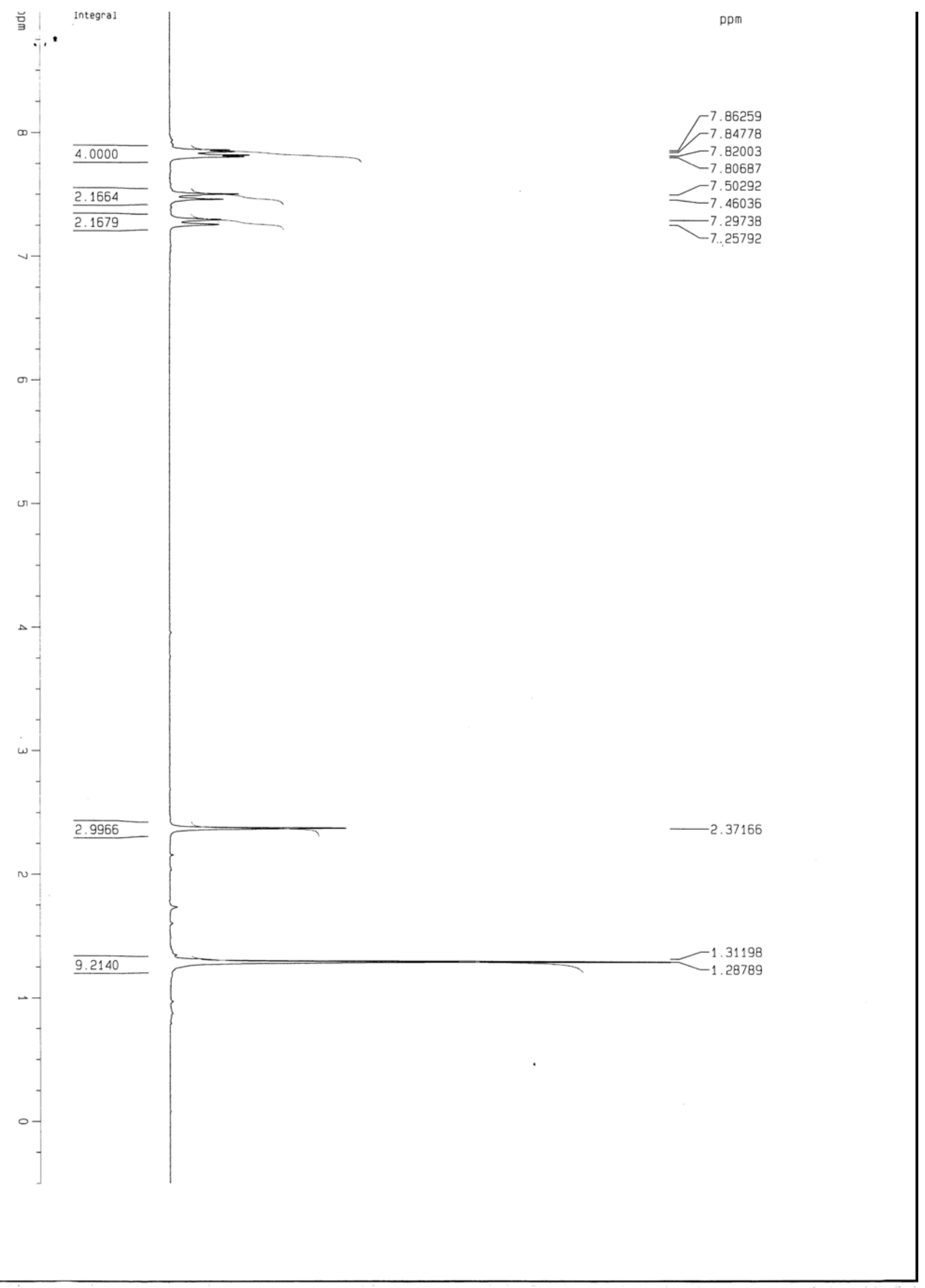


$3 q$

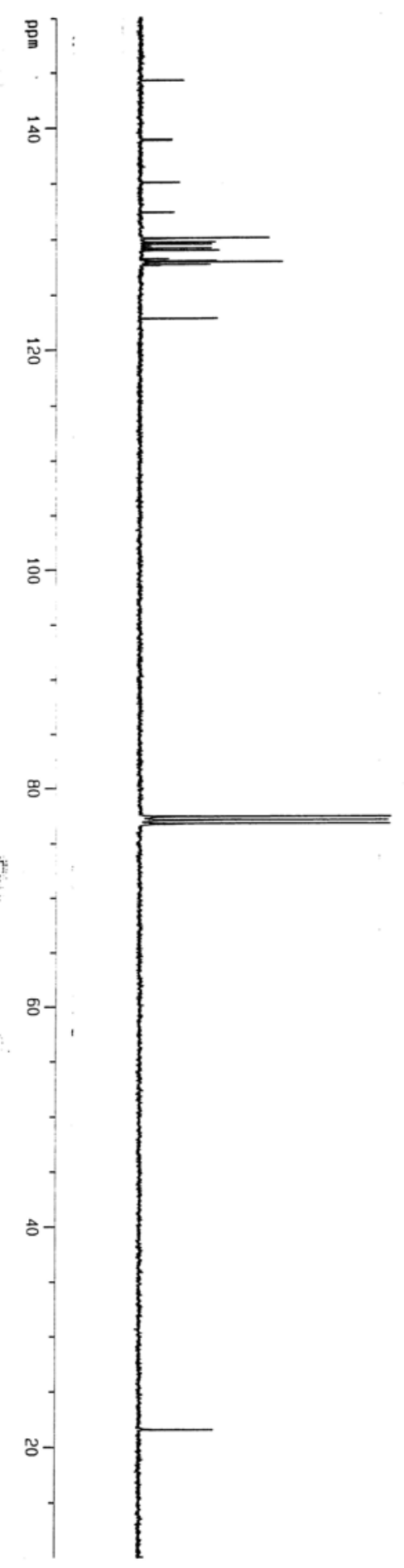<smiles>O=S(=O)(c1ccc([18F])cc1)c1ccc2ccccc2c1</smiles> 


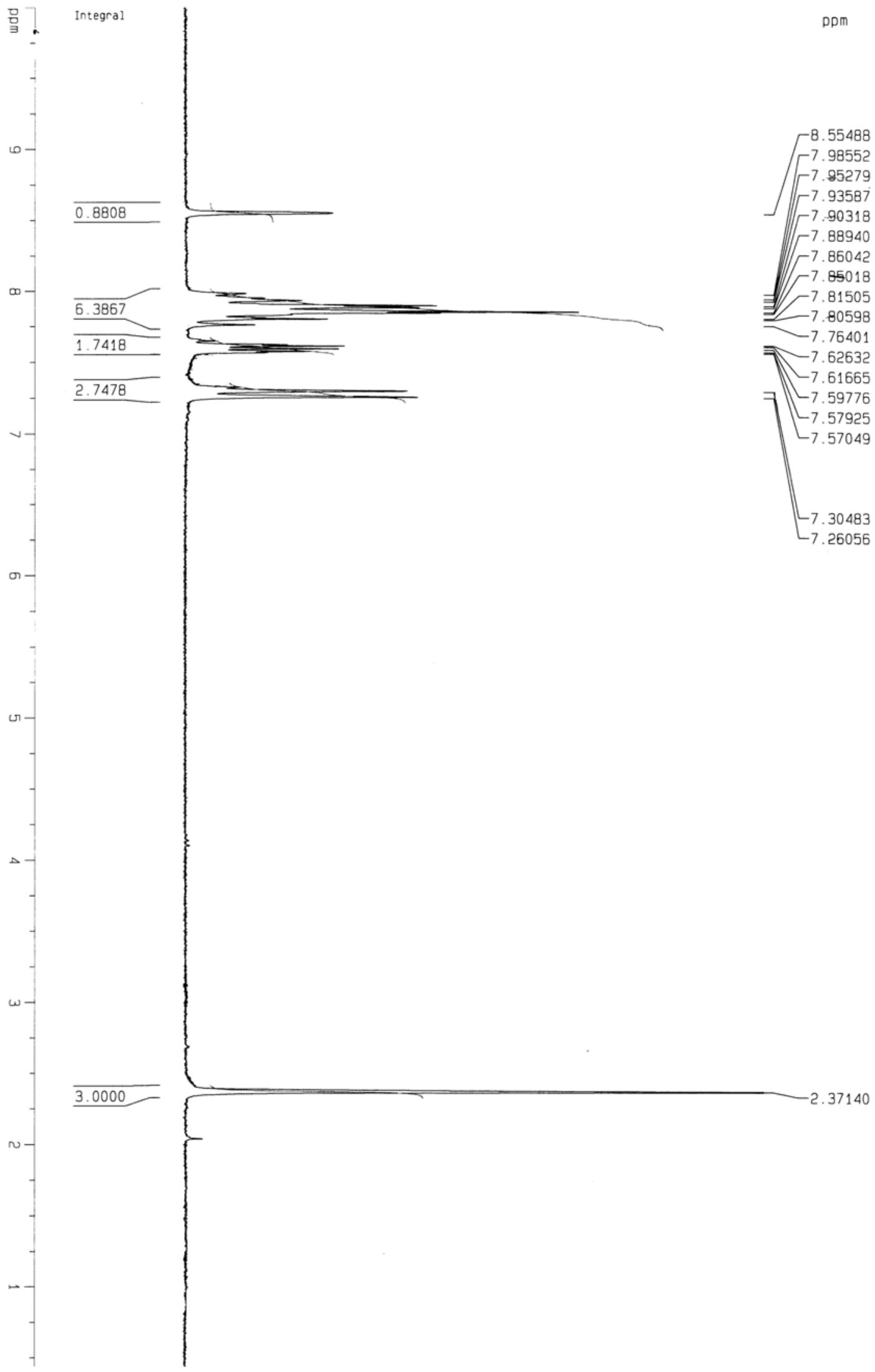


- 21S -

$3 r$
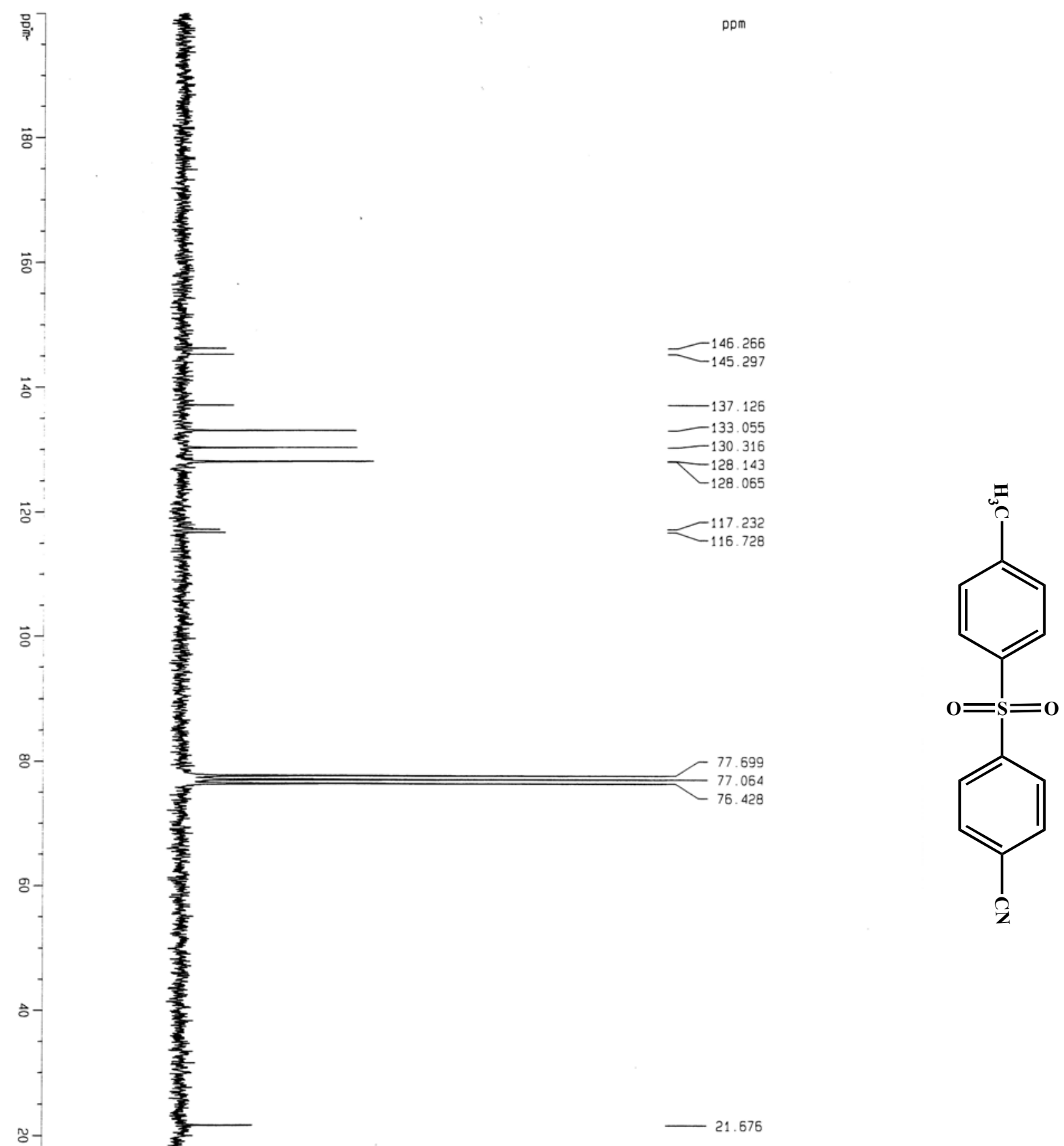
$-22 \mathrm{~S}-$

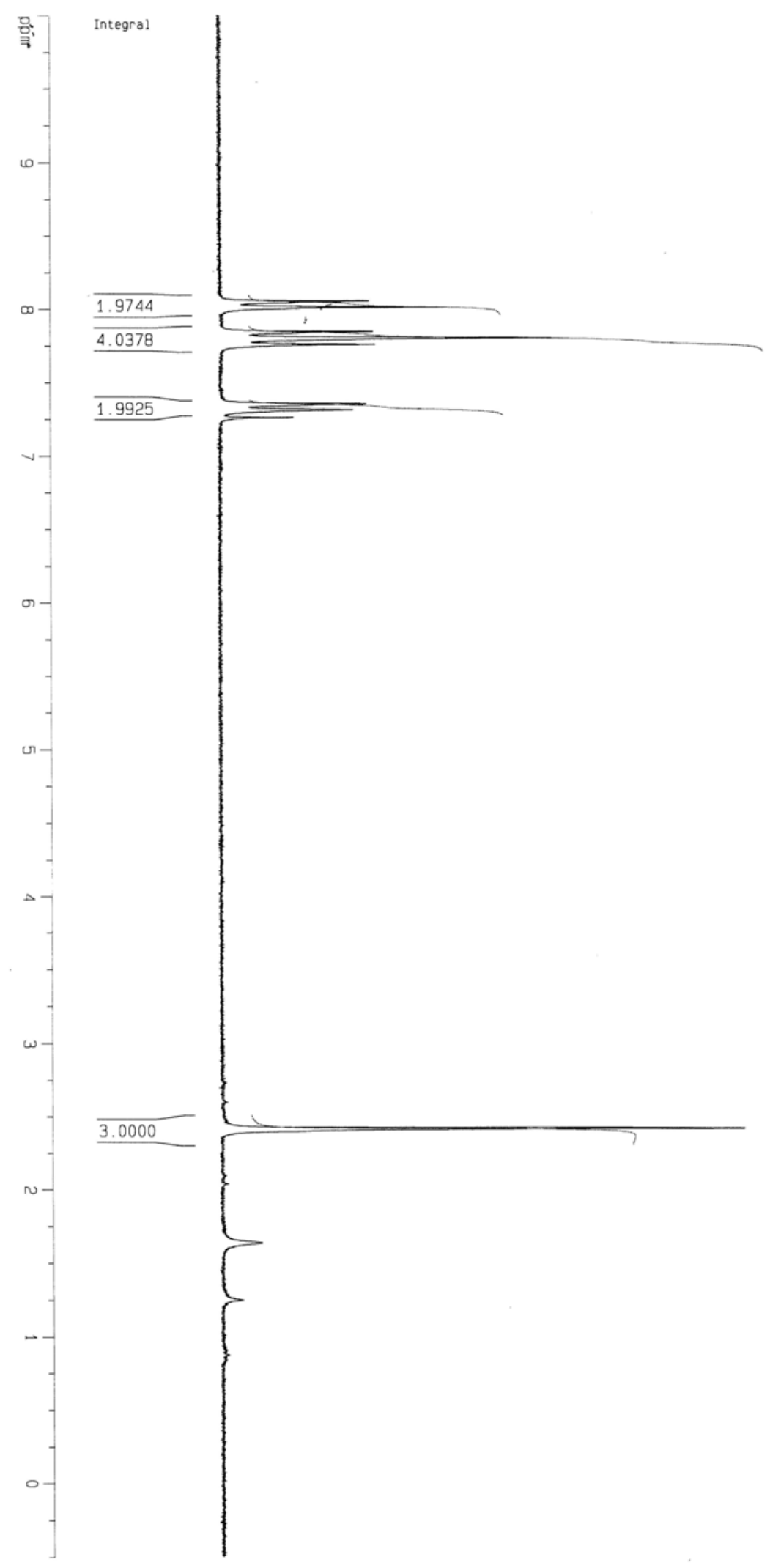

ppm
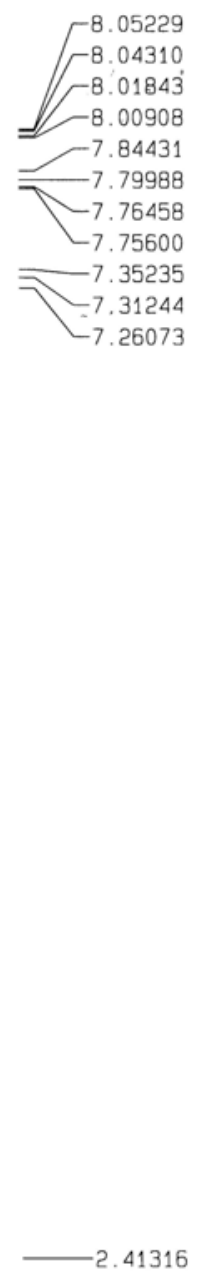
$3 s$

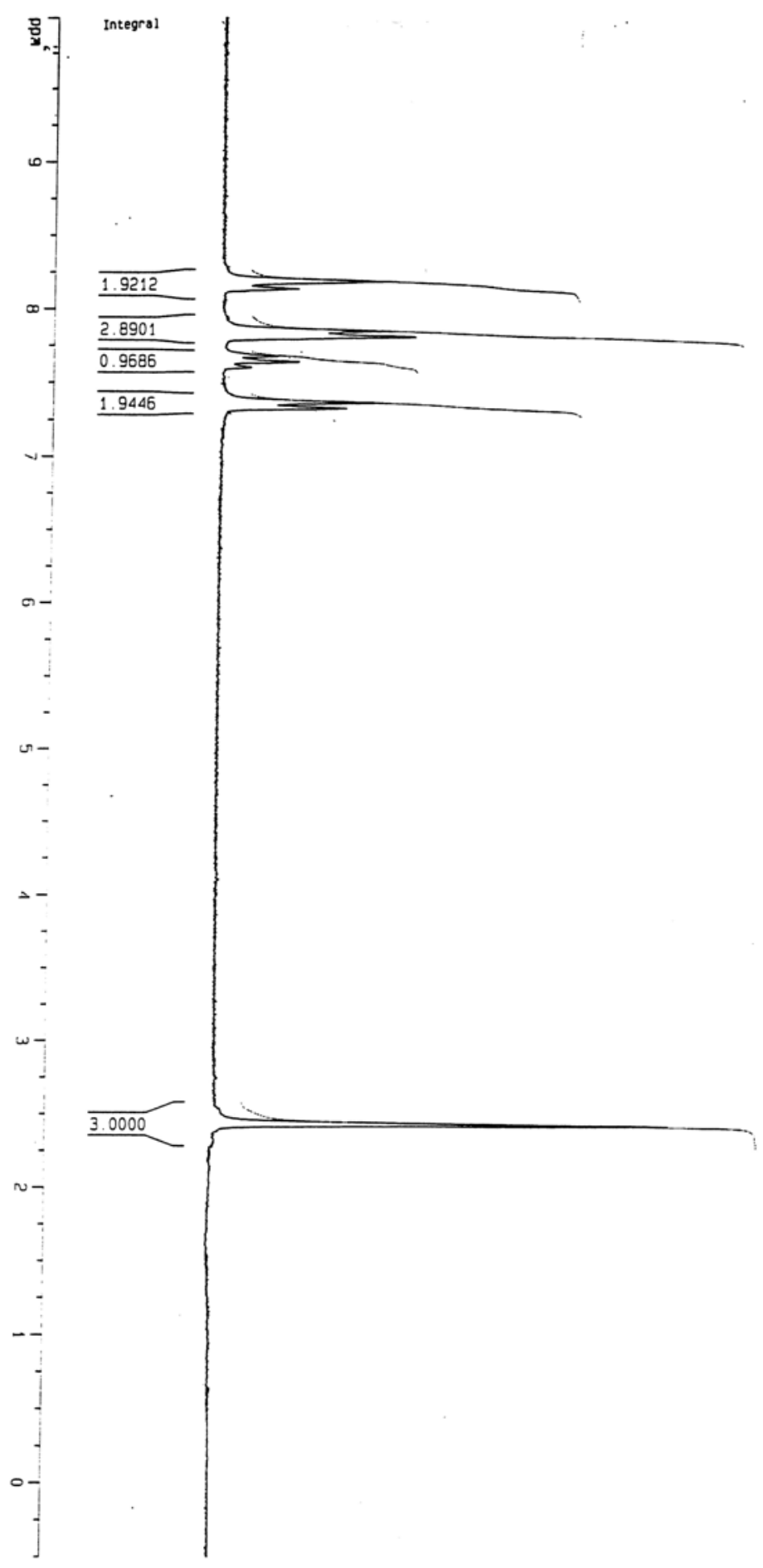

ppm

8.19565

8.14533
-7.86027

7.84145

$-7.82147$

$-7.68999$

$-7.37529$

$-7.33656$

-2. 43131

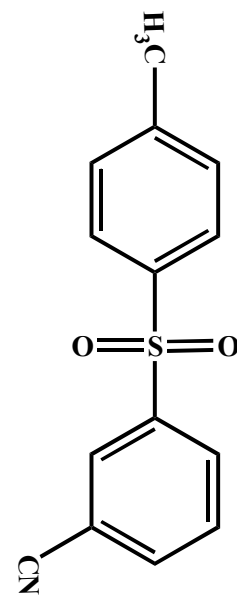



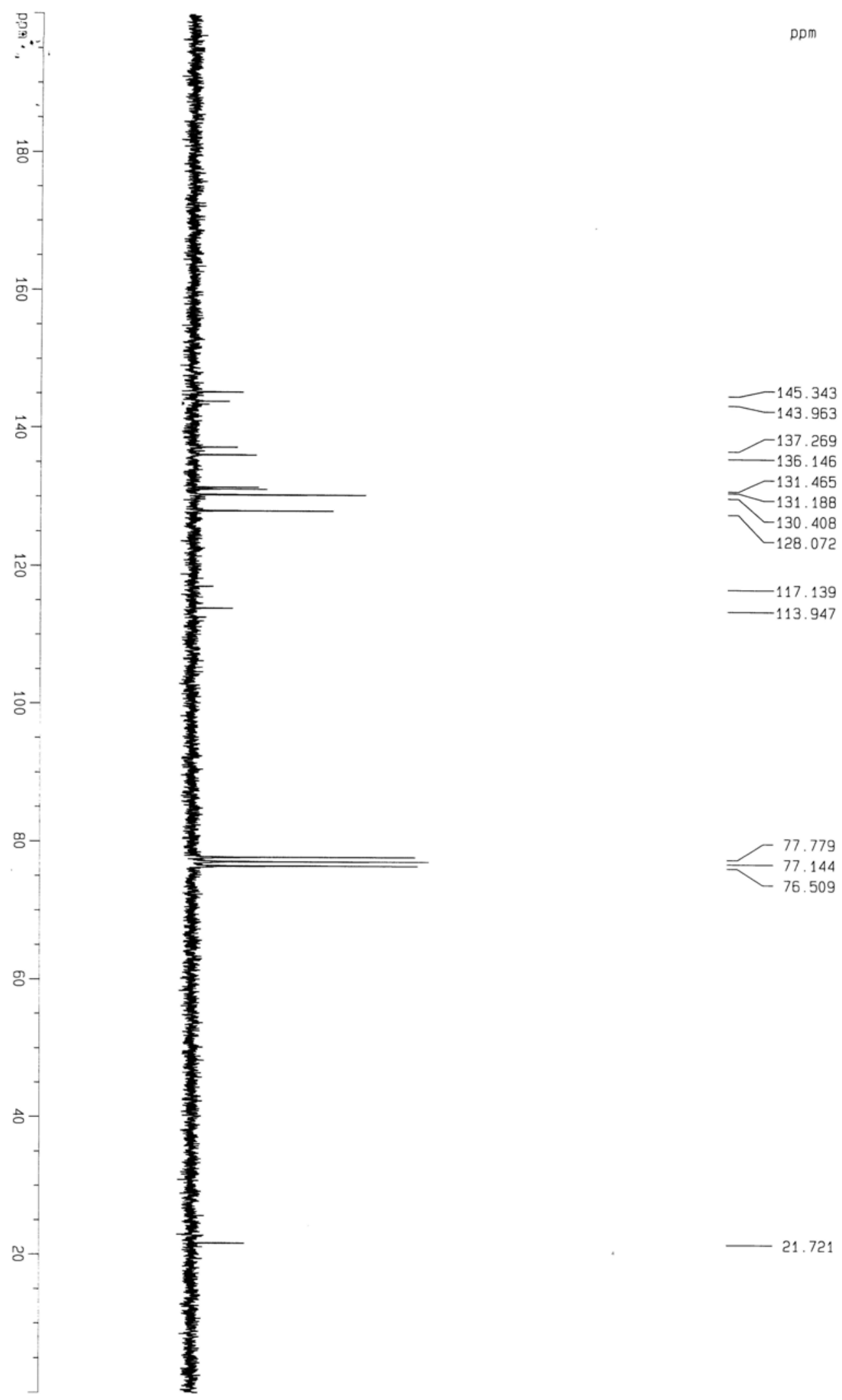

$-76.509$

21.721 


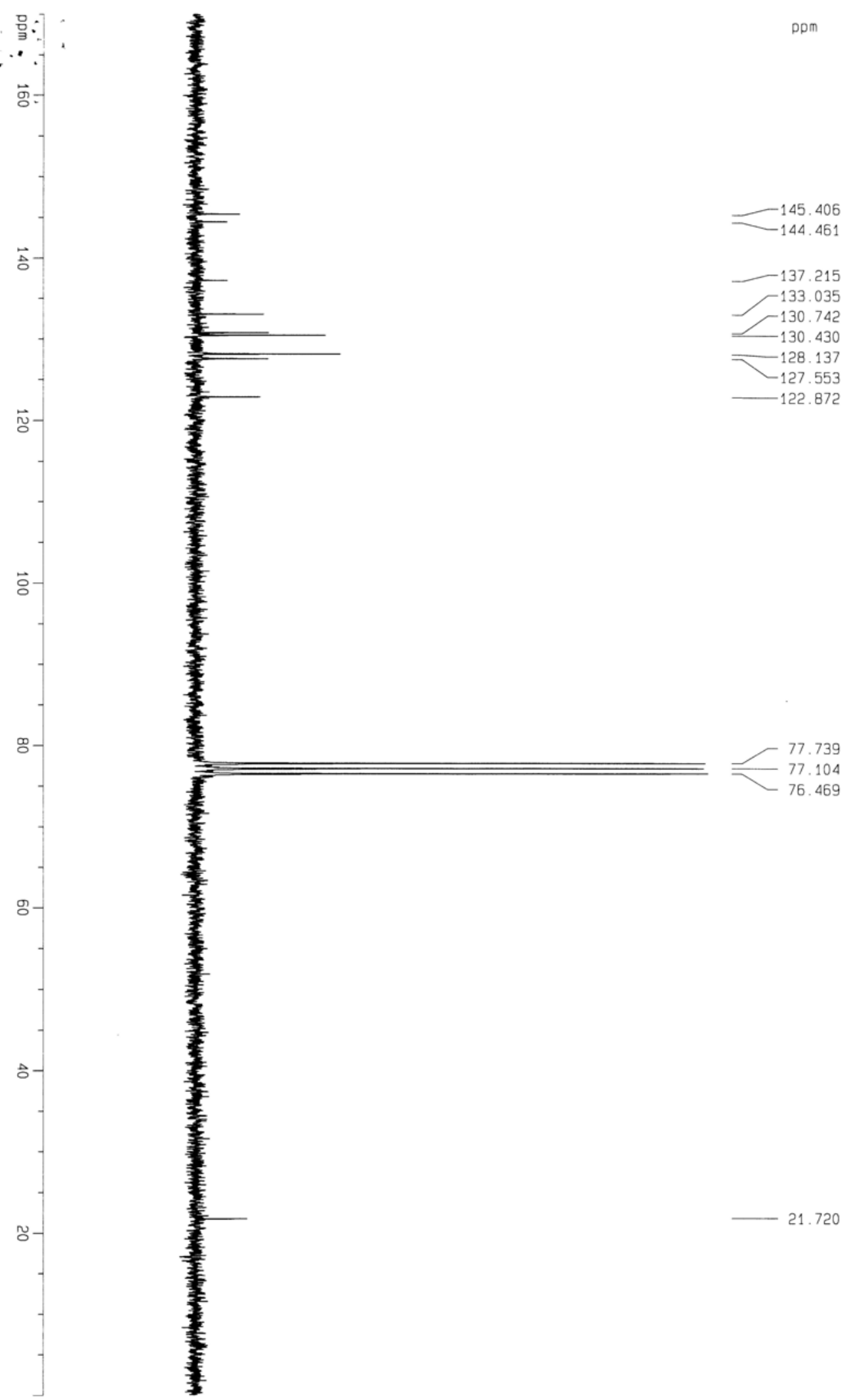




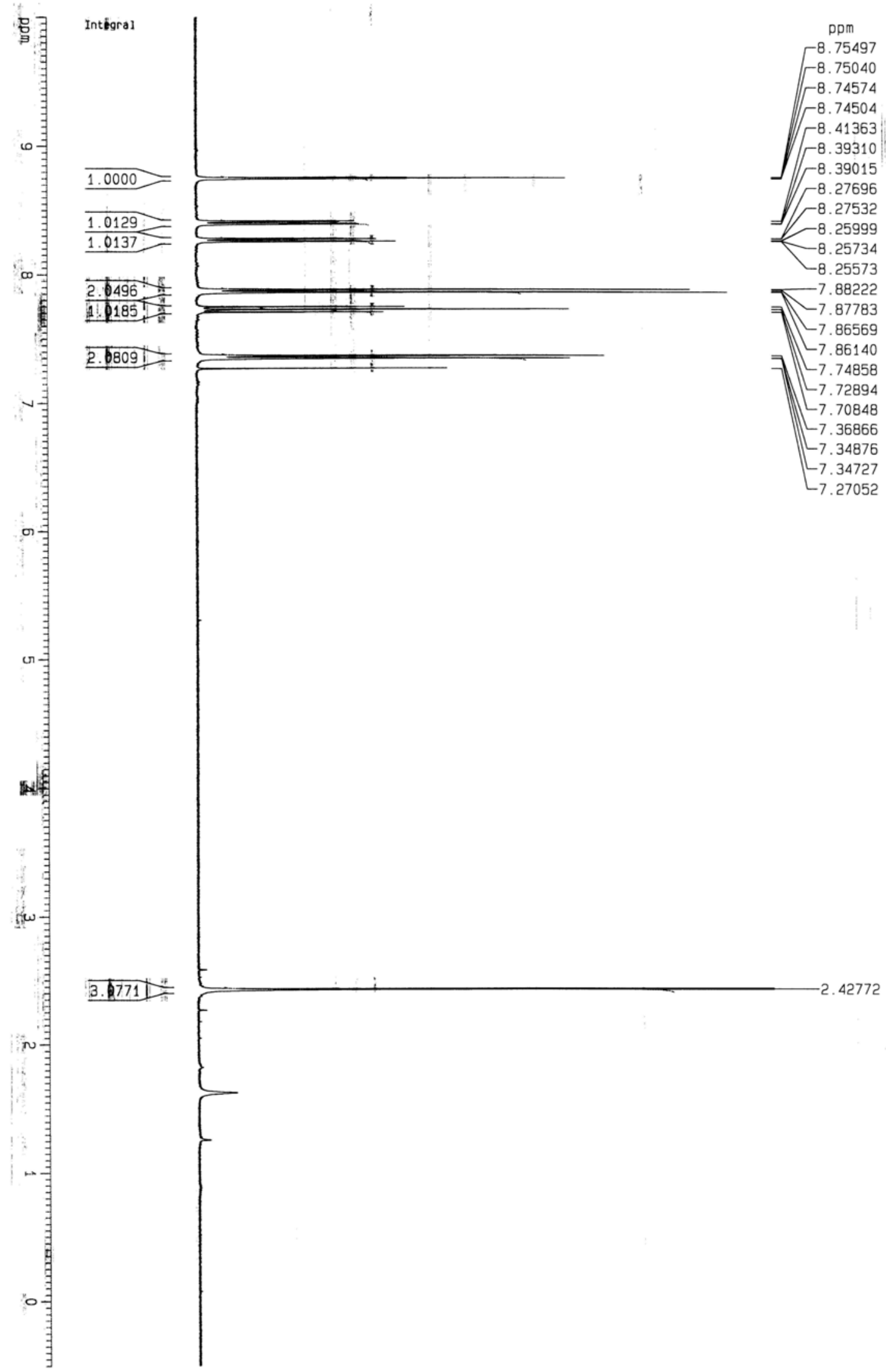


$3 u$
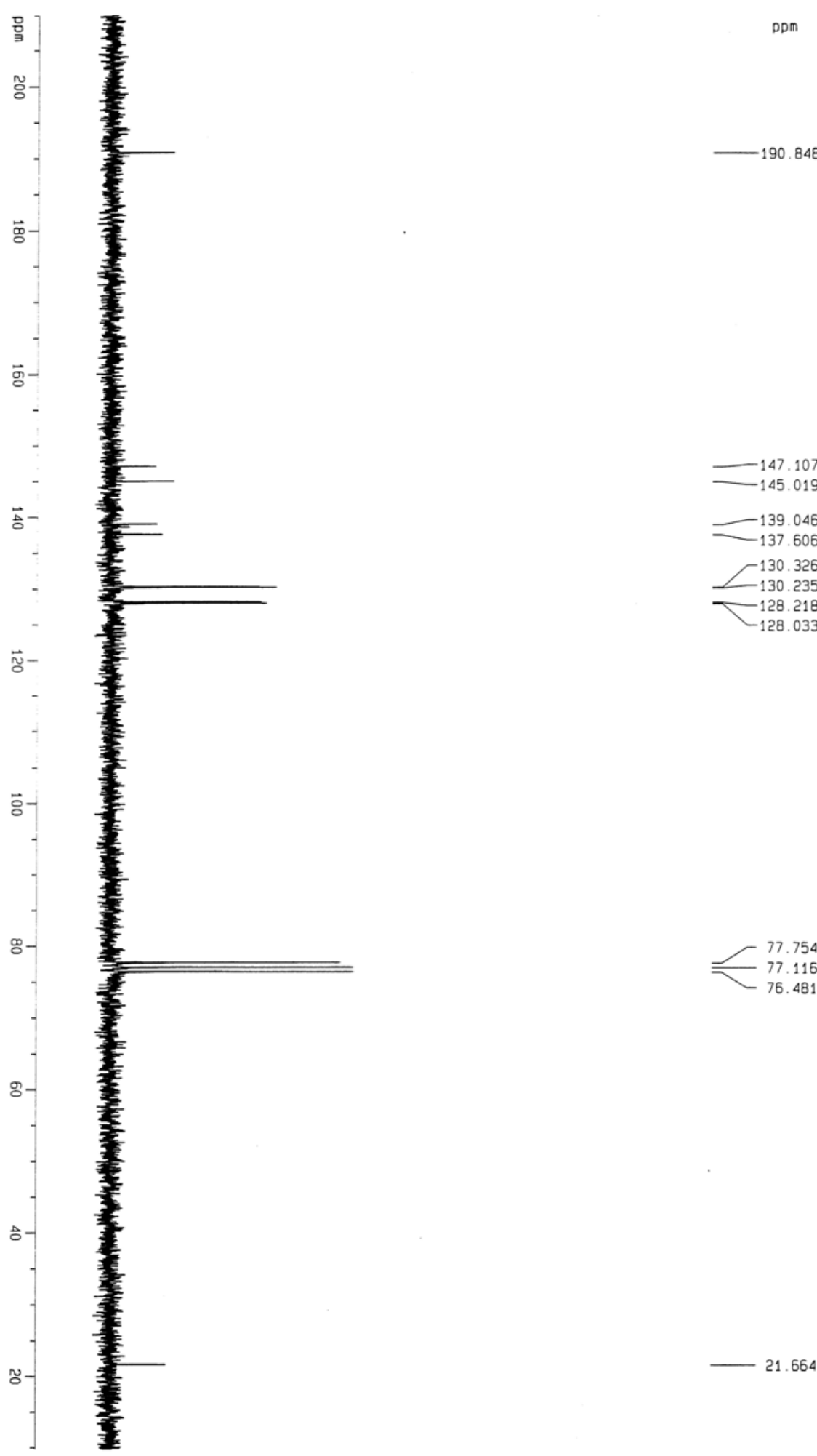

139.046
$-\quad 137.506$

$-130$

-130.235
-128.218

$\sim 128.033$<smiles>COc1ccc(S(=O)(=O)c2ccc([O-])cc2)cc1</smiles>

77.754
$-\quad 77.116$

76.481

ప 
$-28 \mathrm{~S}-$

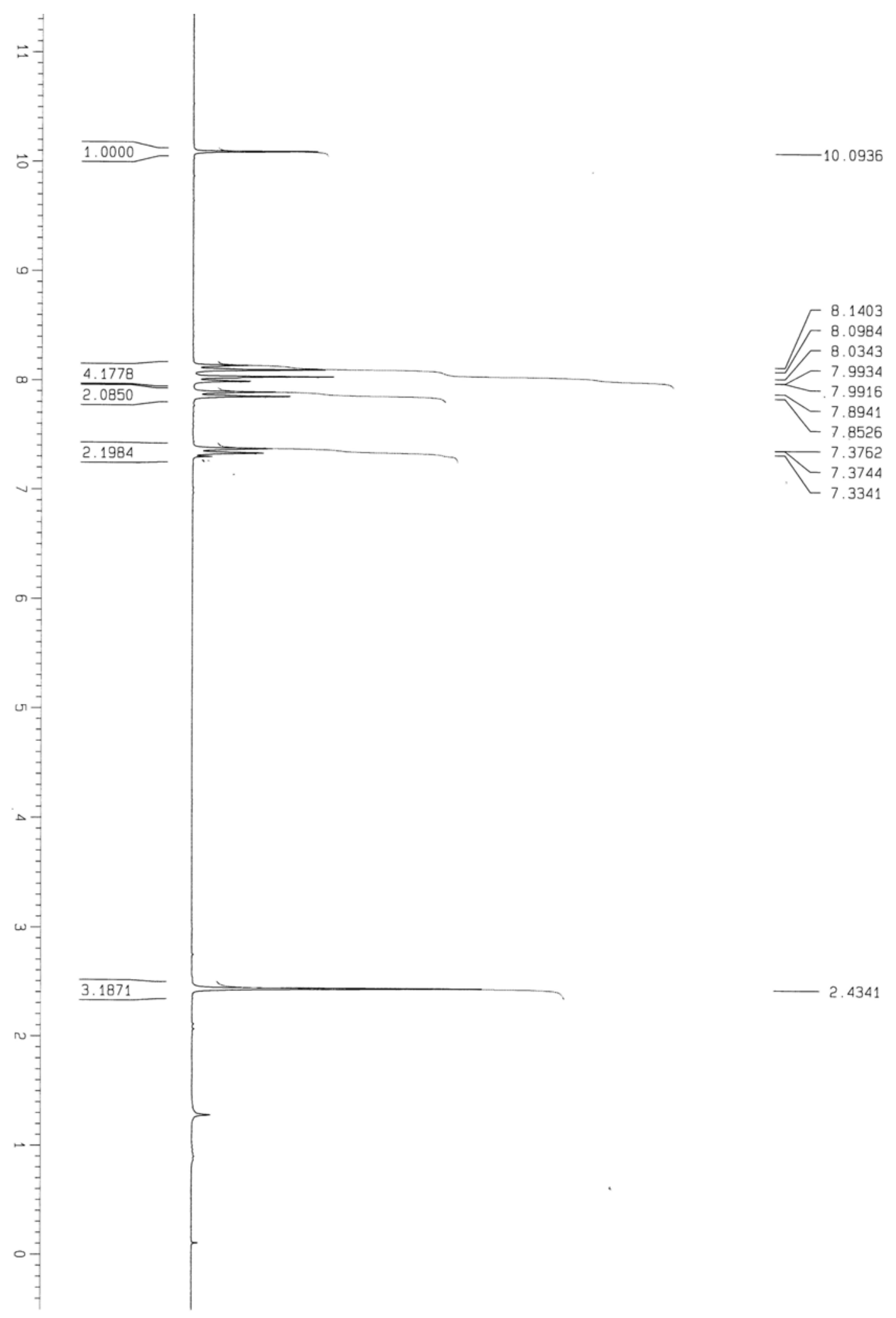


$3 v$

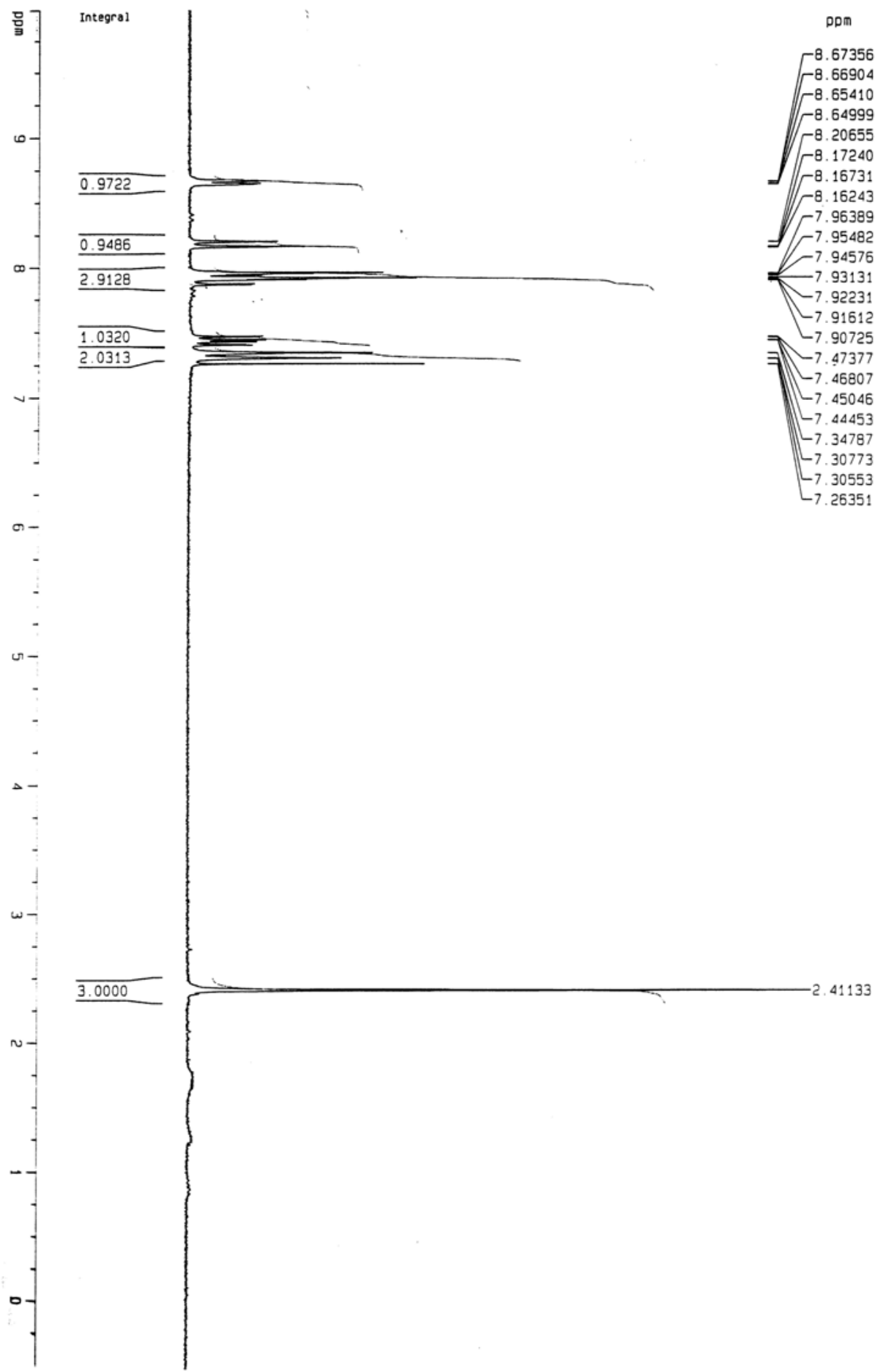




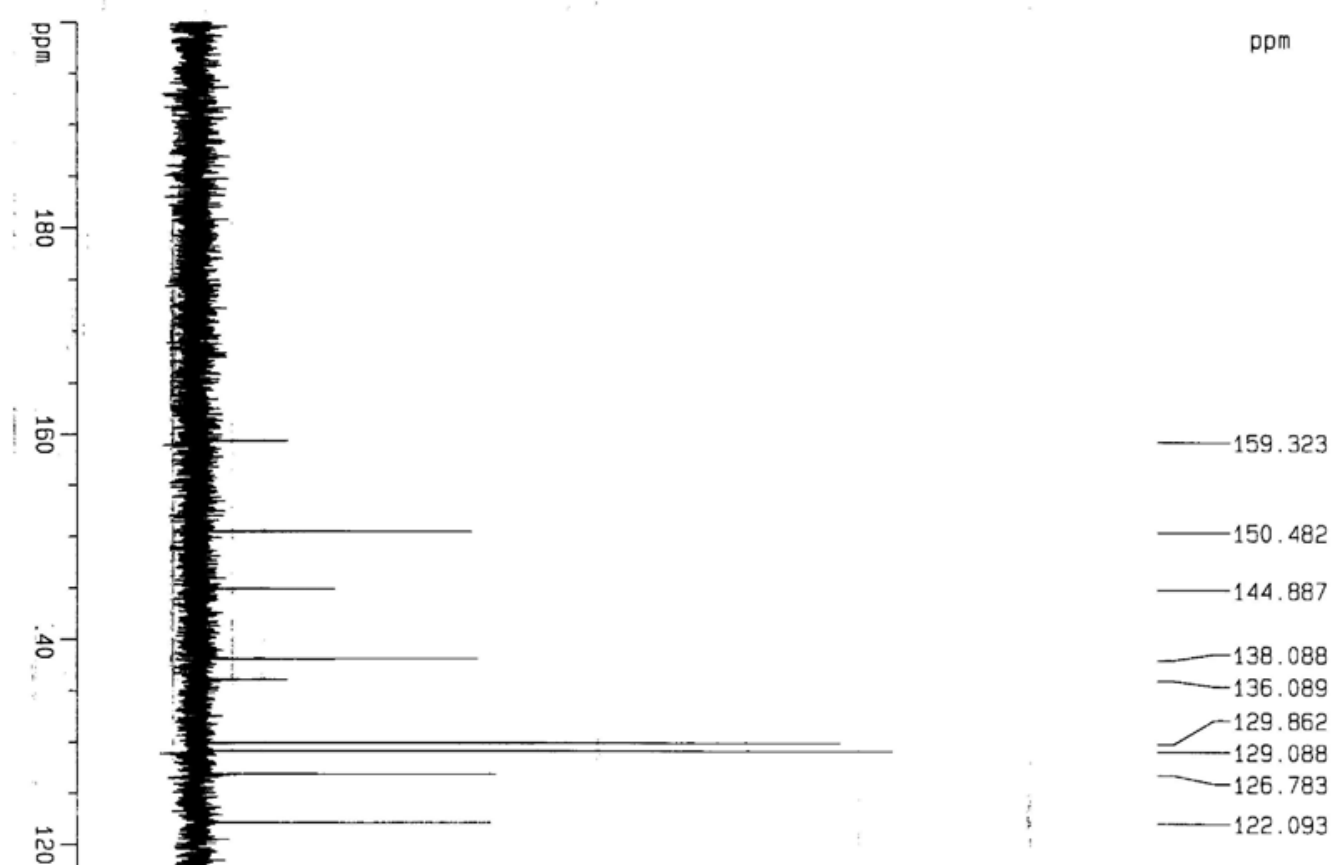

คे

$$
\text { के }
$$


3w
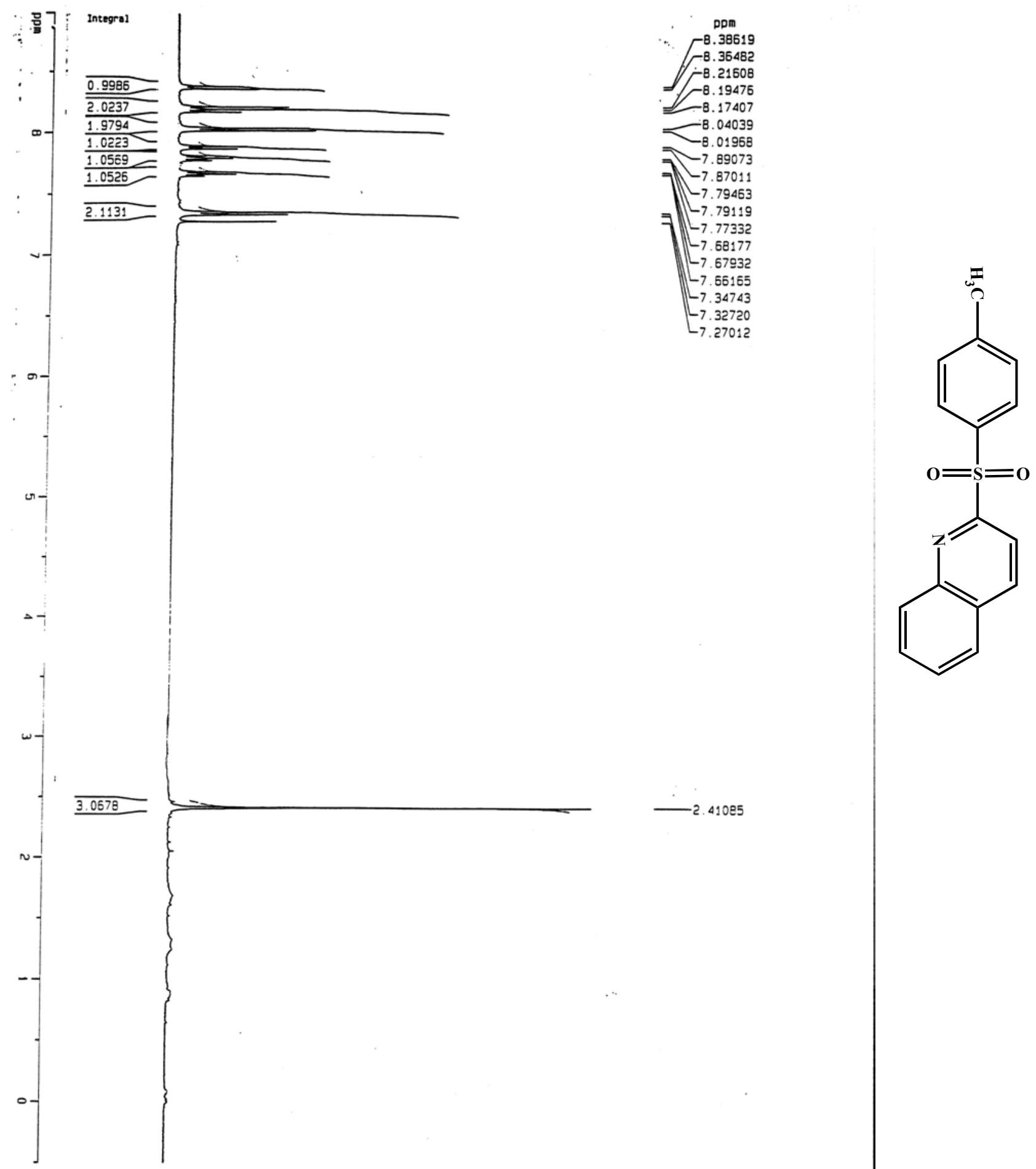

$-2.41085$ 


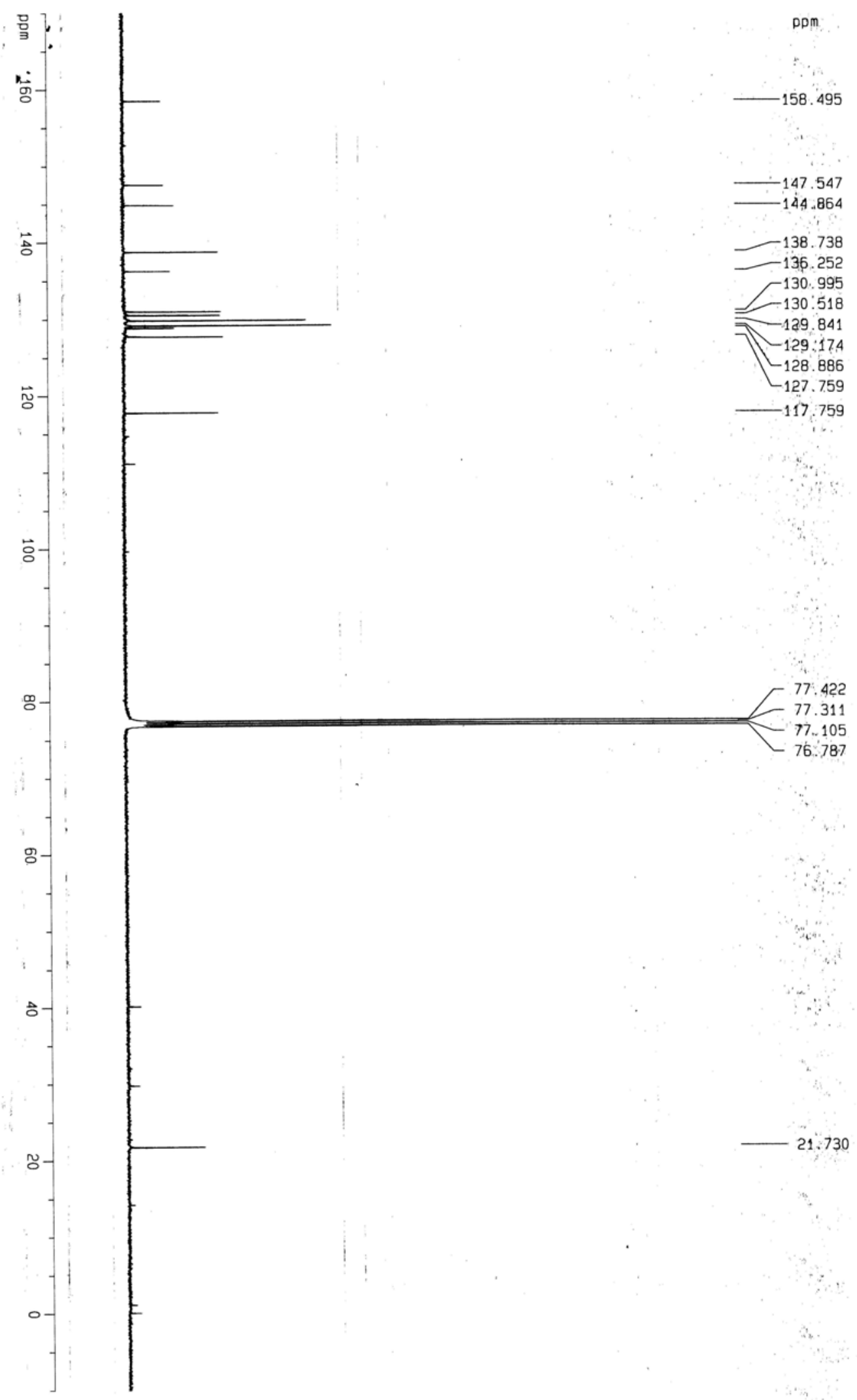


$7 b$
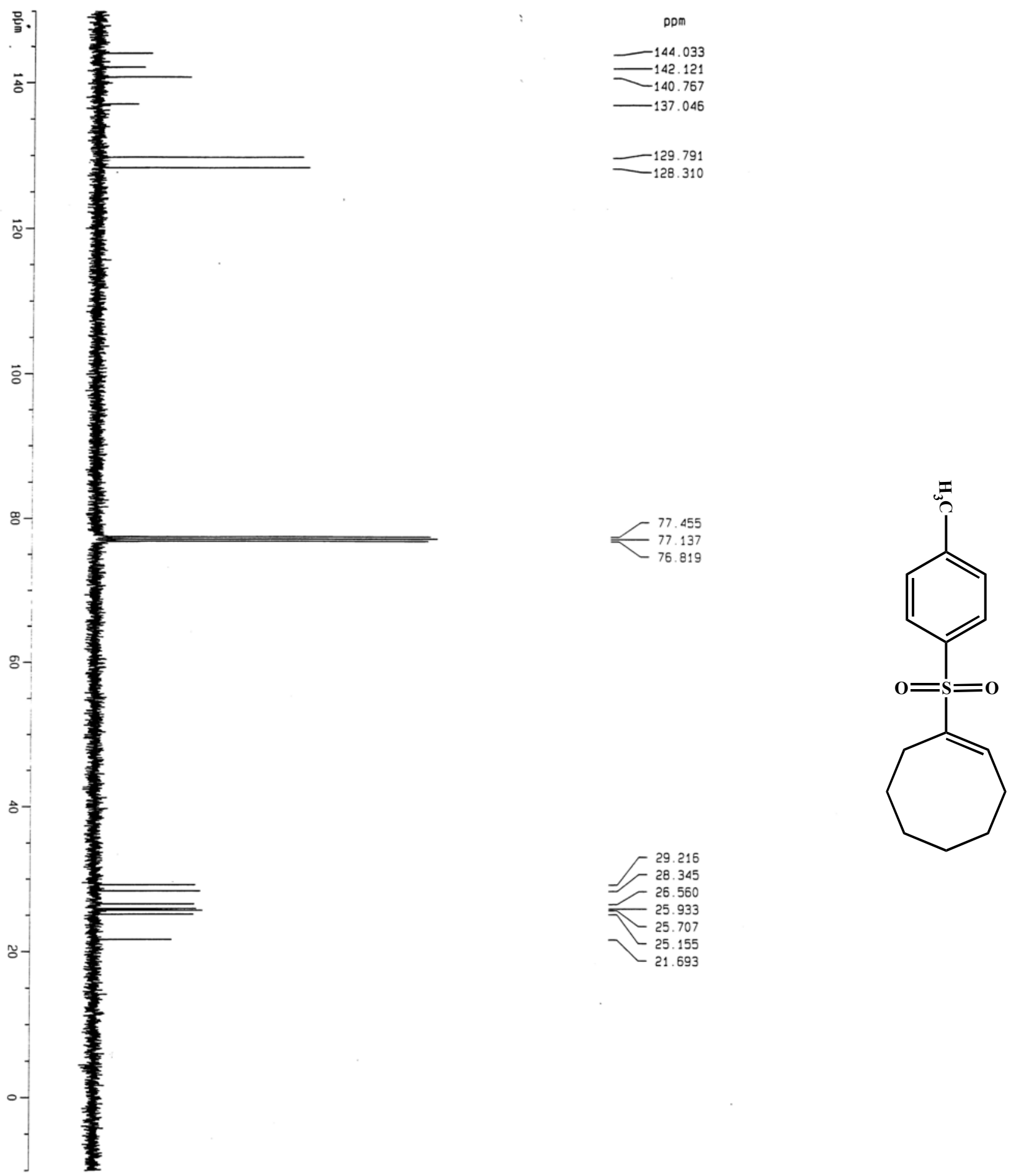

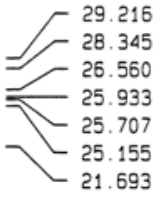


- 34S -

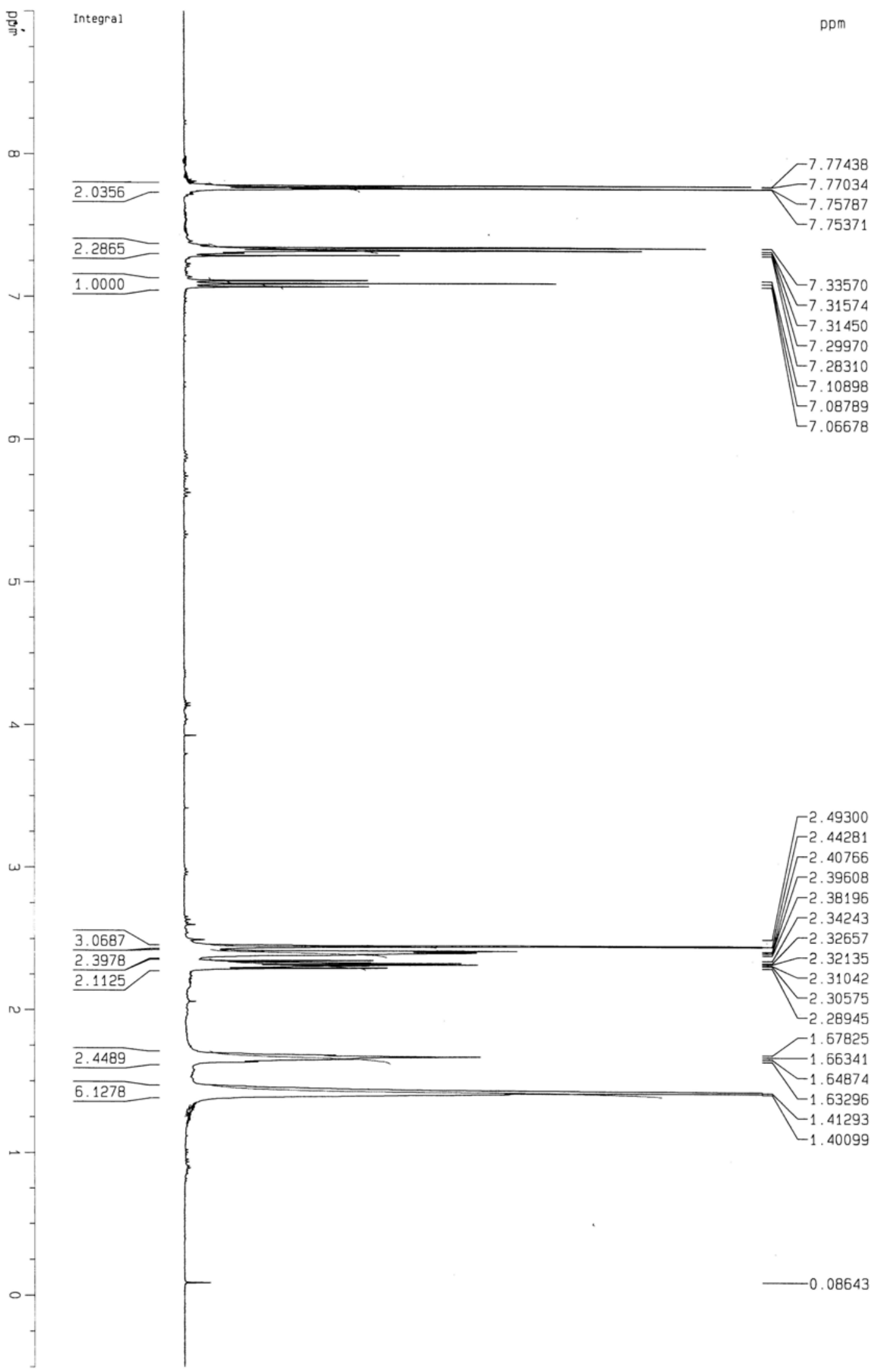


7c
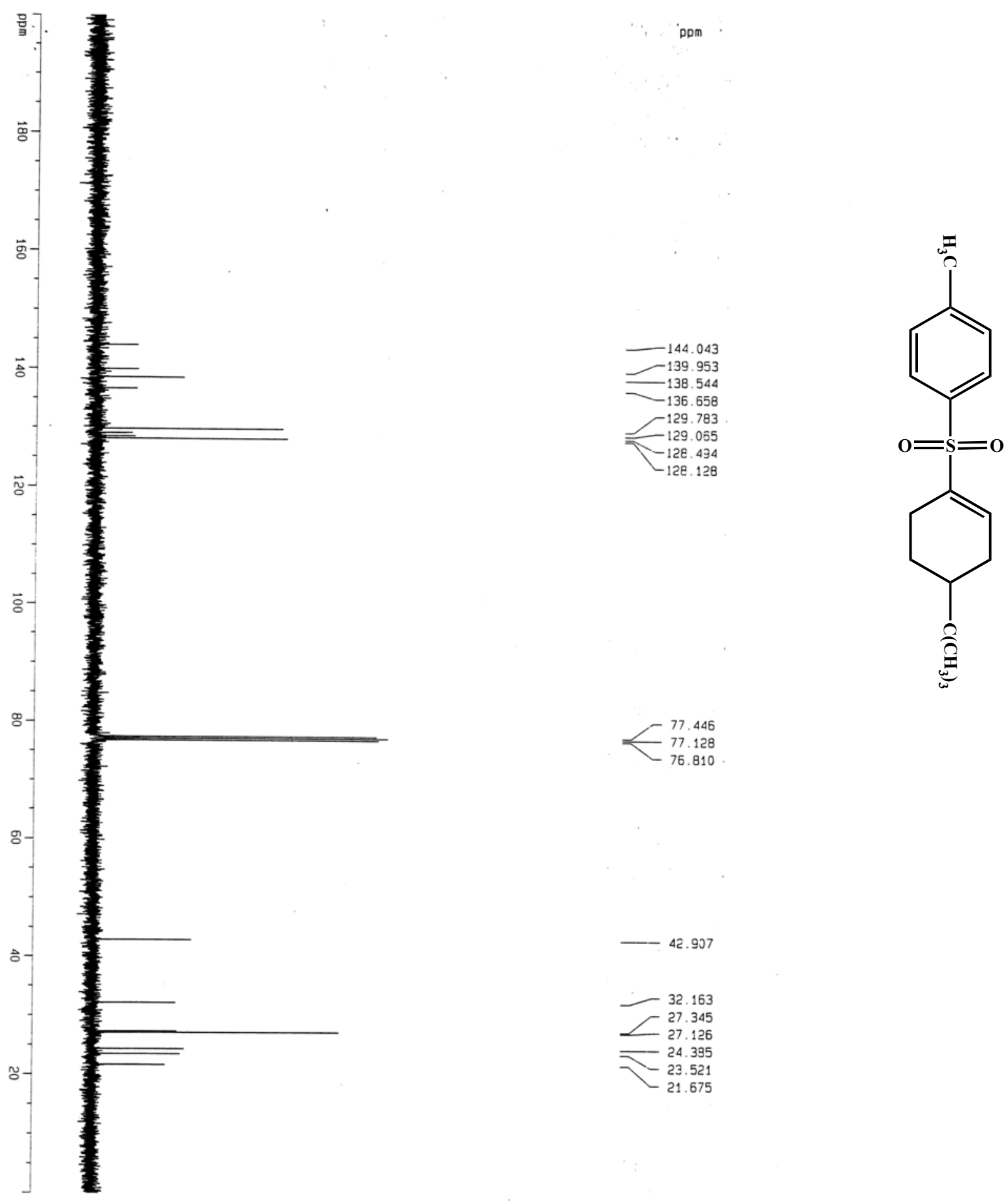

77.446
-77.128

76.810

$-4 \hat{c} .907$

$-32.163$

$-27.345$

27.126

-24.335
$-\quad 23.521$

$-23.521$

$\smile 21.675$ 


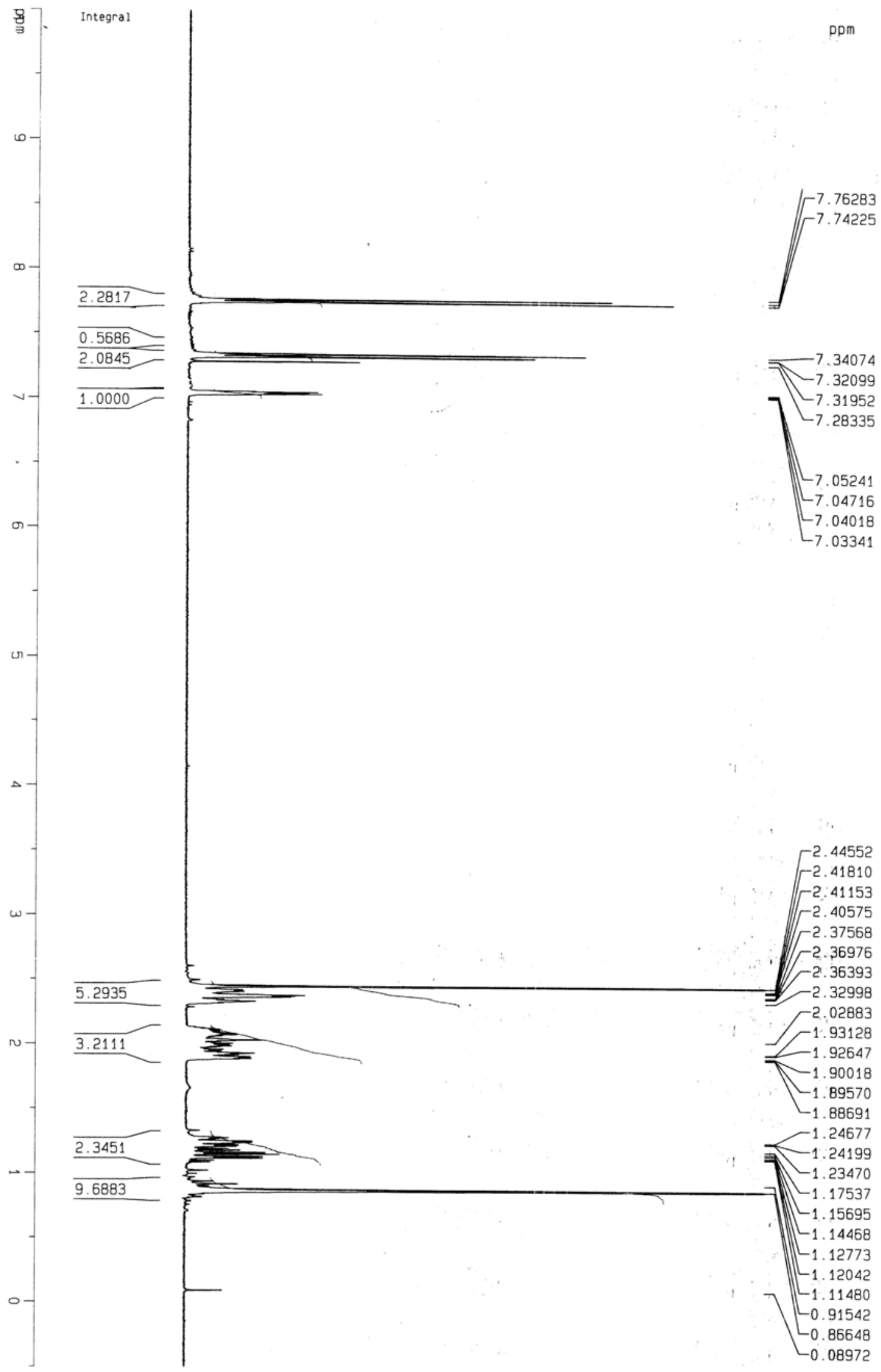


7d
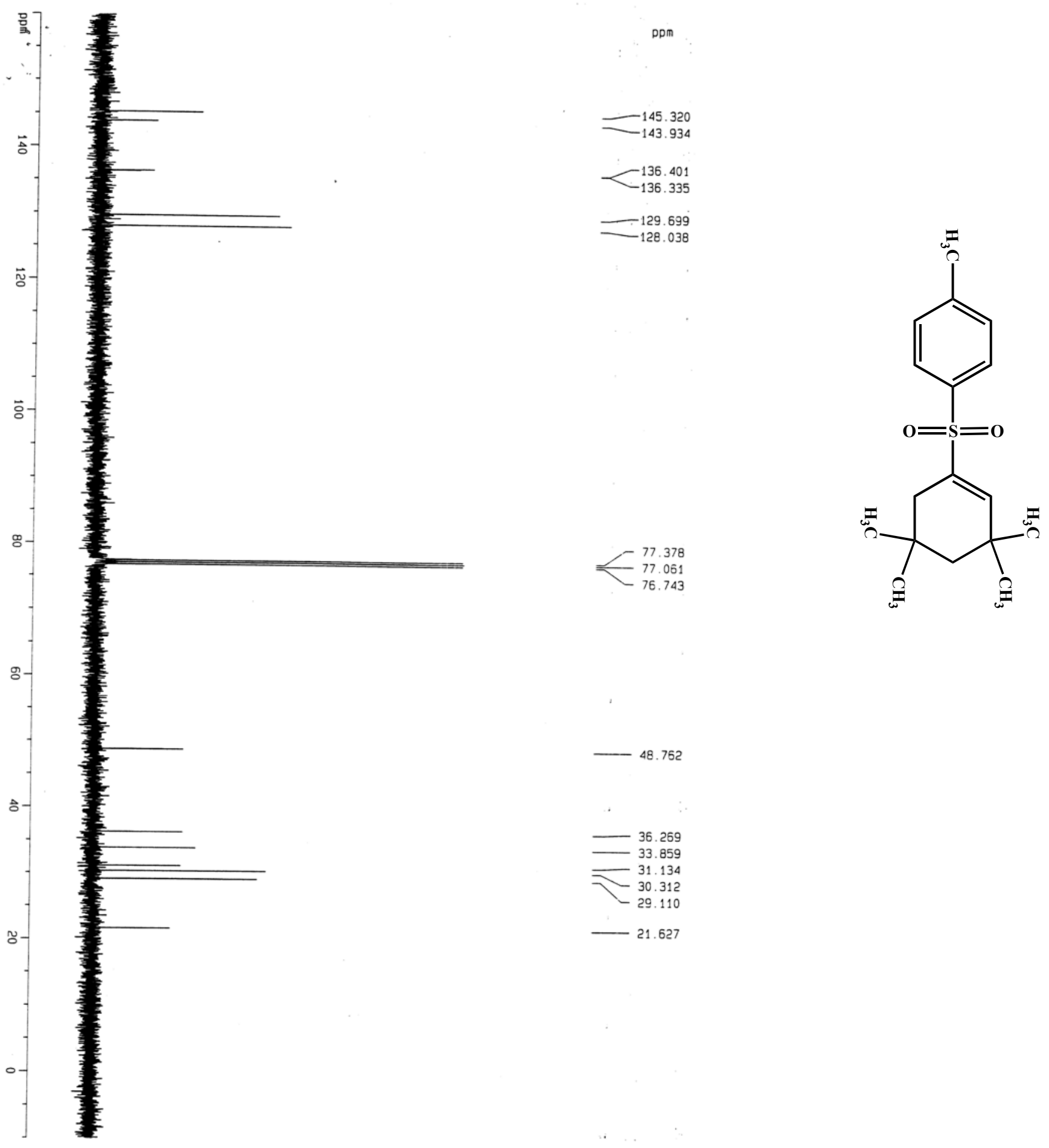

77.378
$\leftarrow$
77.061
76.743

ppm

$-48.762$

- 36.269

33.859

31.134
-30.312

- 30.312

29.110

$-21.627$ 
- 38S -

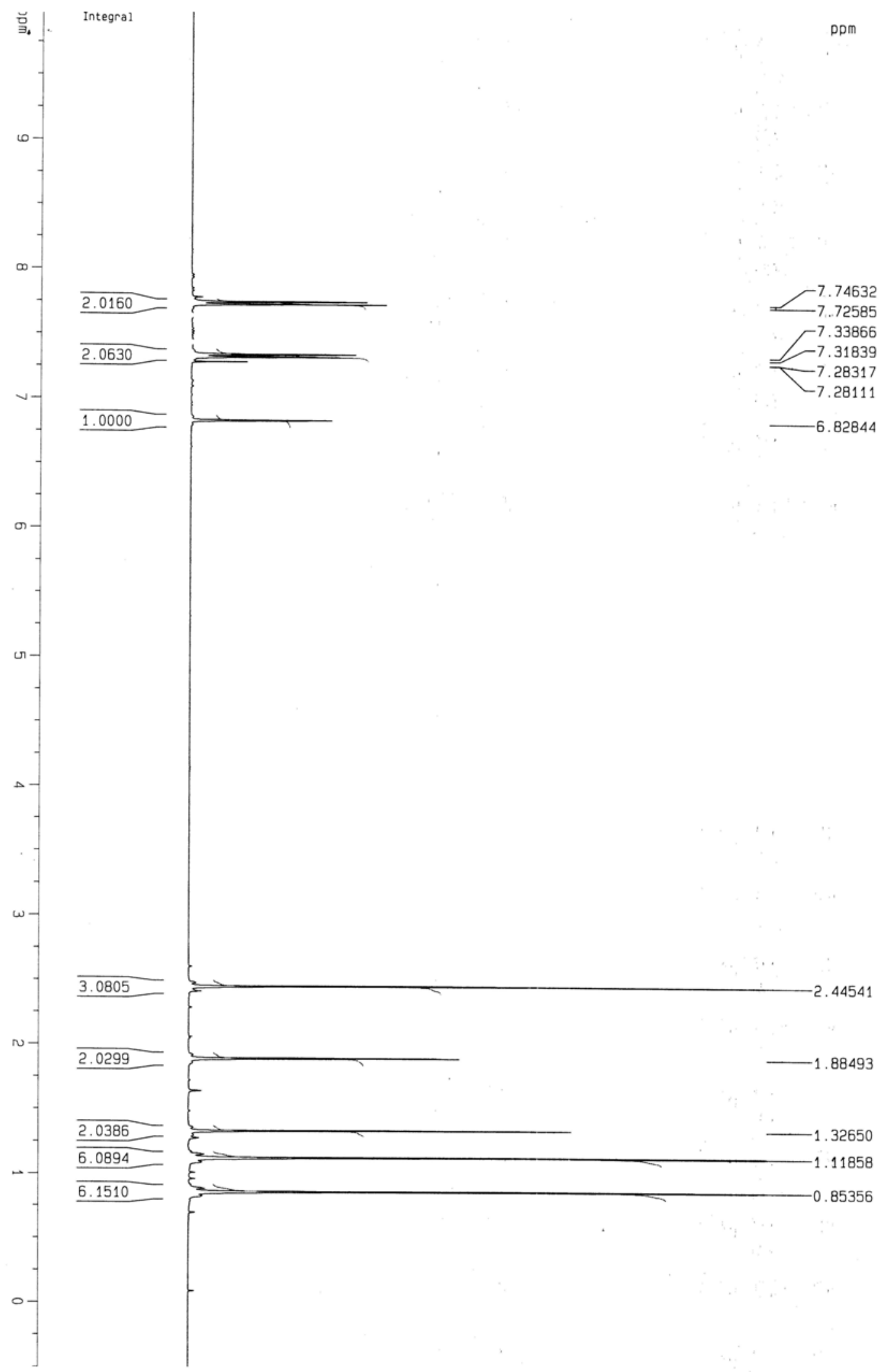



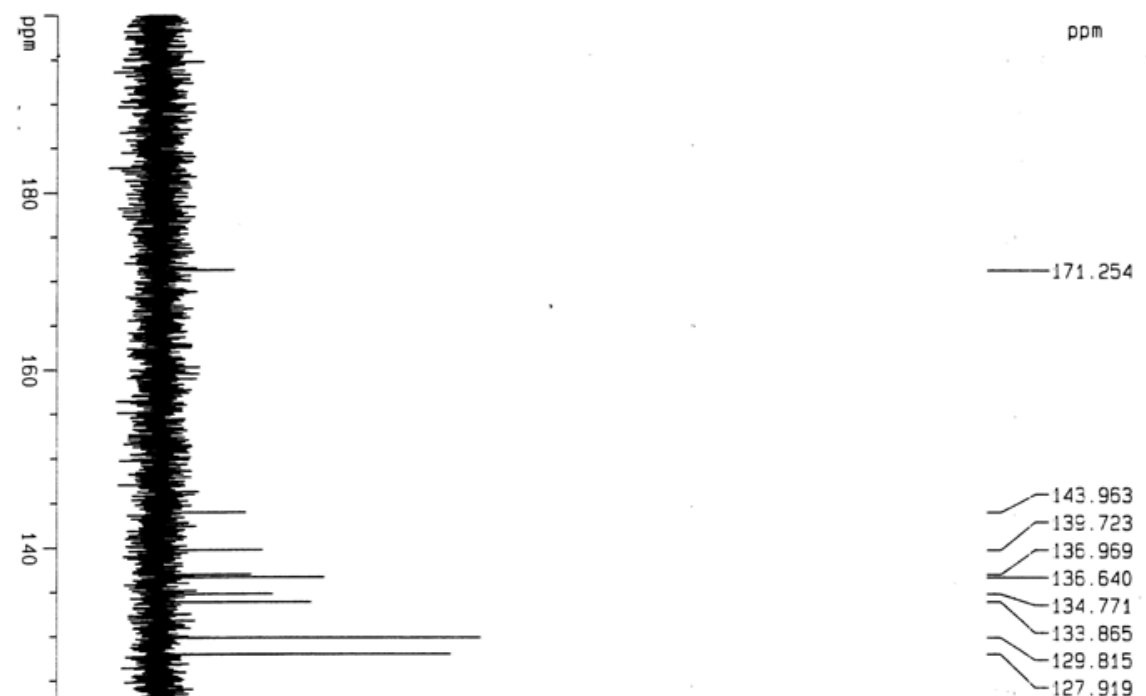

픙-

82.596

$-77.429$

$\begin{array}{r}-77.439 \\ -77.112 \\ \hline\end{array}$

$-76.794$

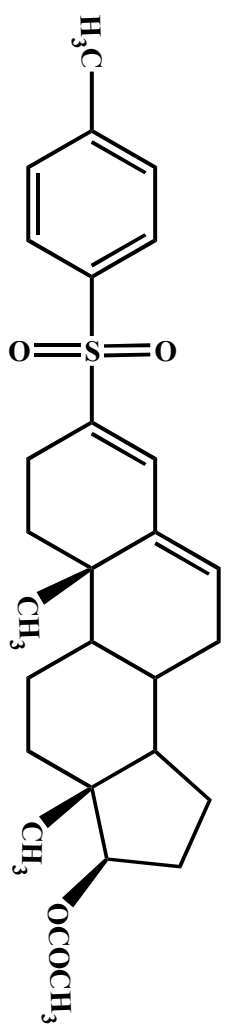

$-51.162$

-4.809
$-\quad 4.591$

- 42.591

- 36.676

- 34.856

- 33.375

31.958
-31.505

$-31.505$

$\begin{array}{r}-27.565 \\ \hline\end{array}$

23.525
$-\quad-51.580$

$=21.680$

$\simeq 21.243$

ᄂ 20.897

L 20.675

$\begin{array}{r}-12.119 \\ \hline\end{array}$ 


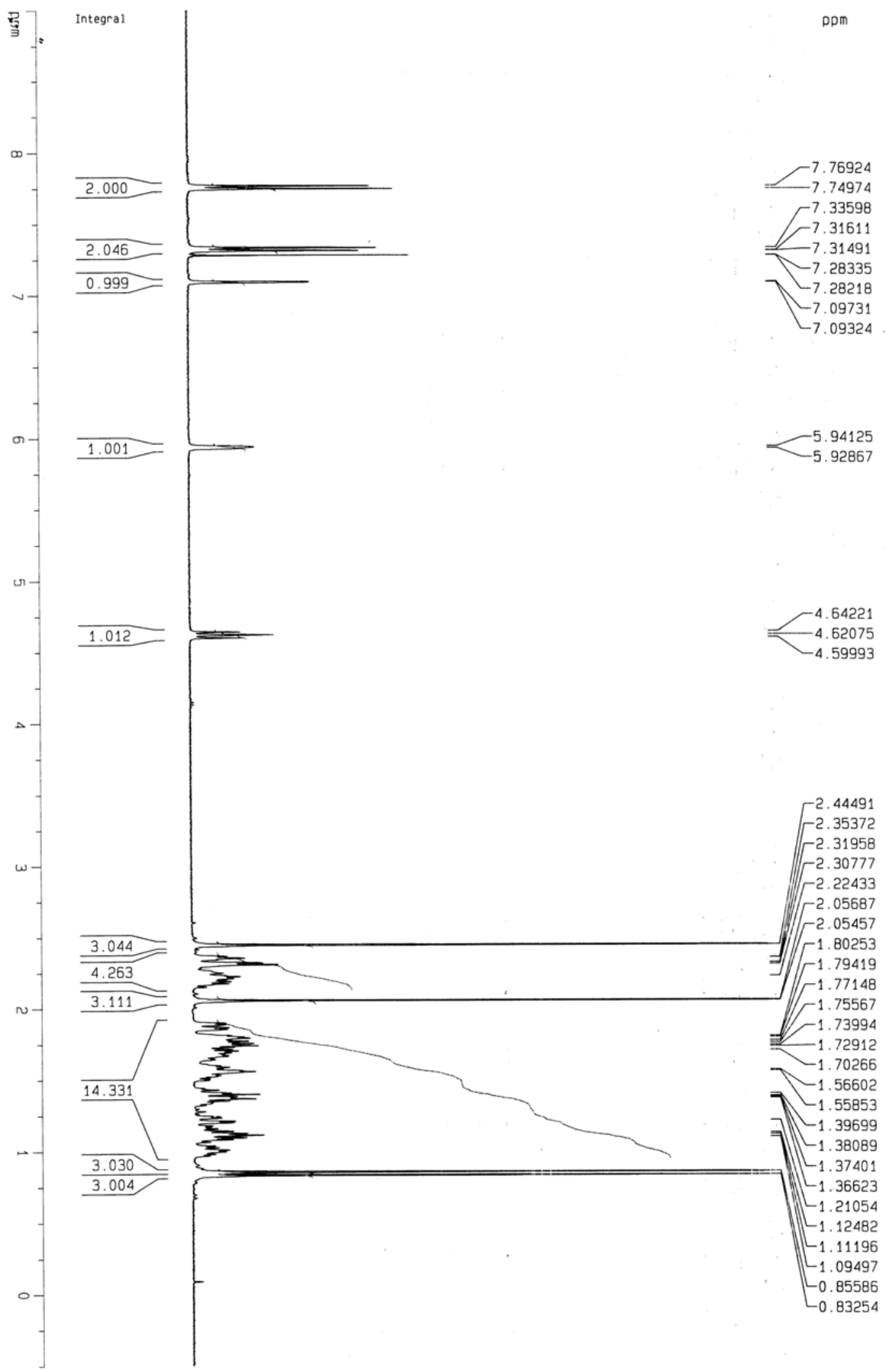



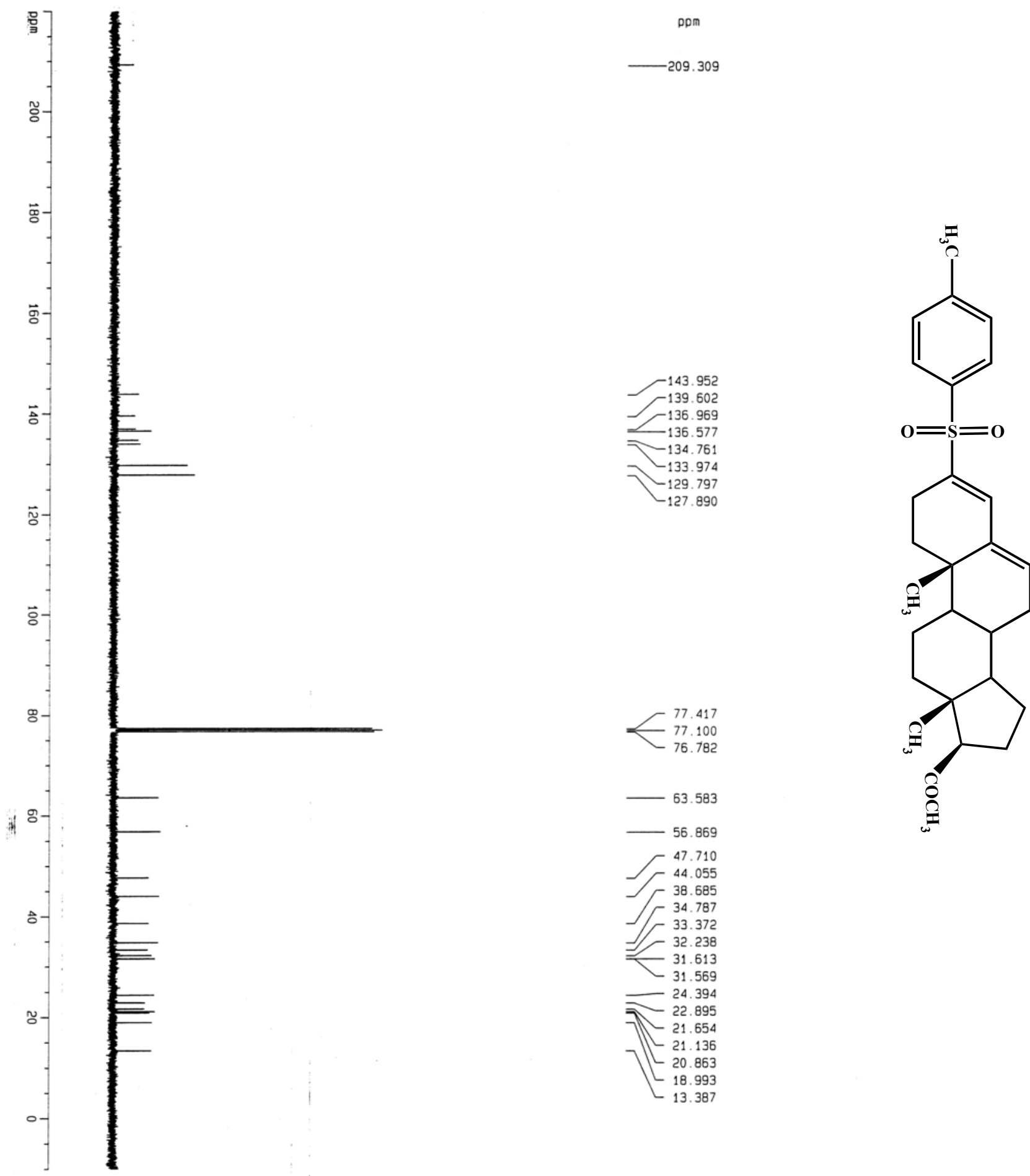

$-56.869$

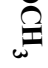

C 47.710

$\tau^{44.055}$

$\tau^{34.78}$

- 33.372

(3).

31.613
-31.569

$\begin{array}{r}-31.569 \\ \hline\end{array}$

- 24.394

- 22.895

$\simeq 21.654$

$-21.136$

$-20.863$

- 18.993

- 13. 387 


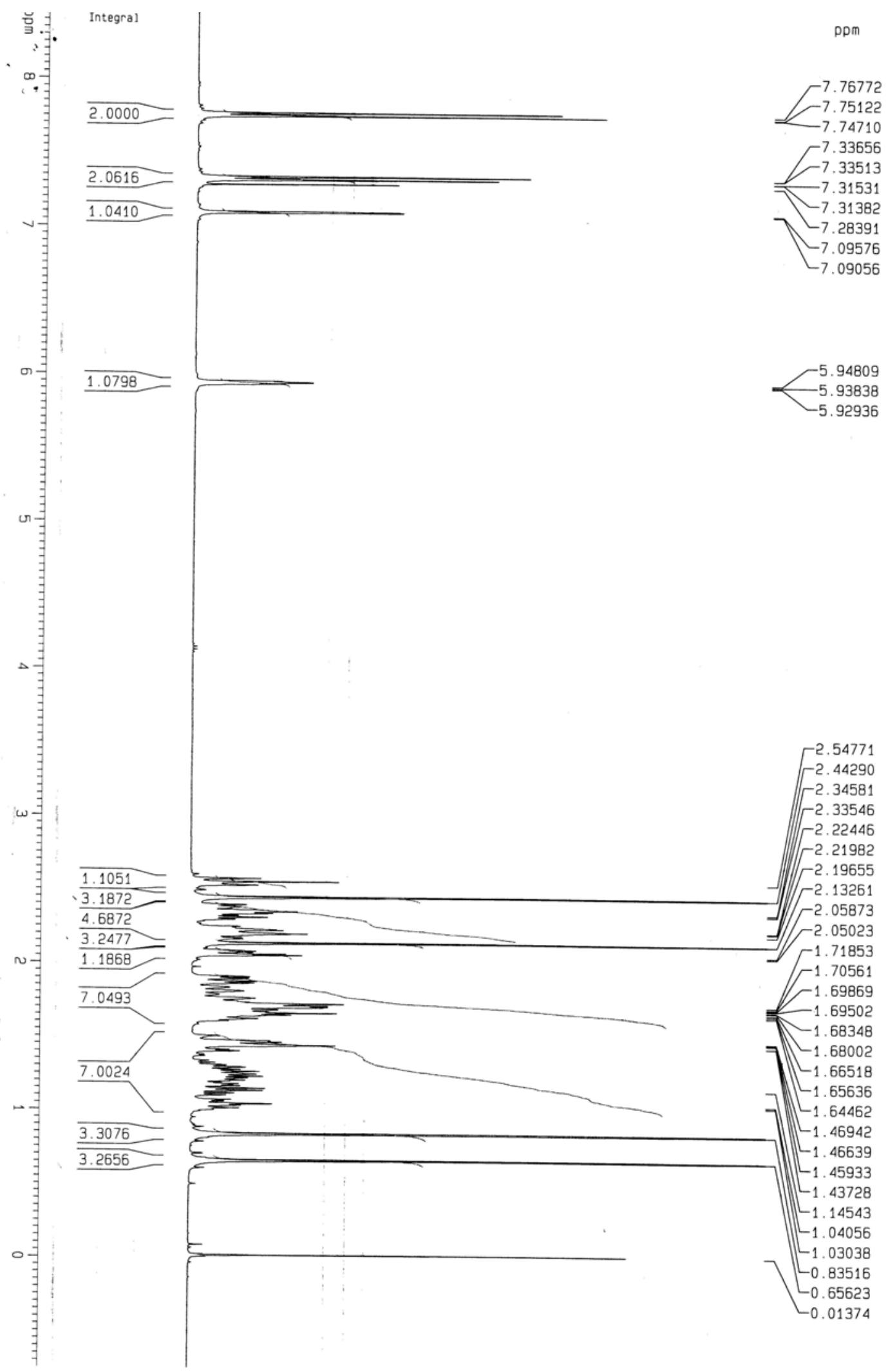

